\title{
Network Capacity under Traffic Symmetry: Wireline and Wireless Networks
}

\author{
Sudeep Kamath*, Sreeram Kannan ${ }^{\dagger}$, Pramod Viswanath ${ }^{\dagger}$ \\ *EECS Department, University of California, Berkeley, \\ \{sudeep\}@eecs.berkeley.edu \\ ${ }^{\dagger}$ Electrical and Computer Engineering, University of Illinois Urbana-Champaign, \\ $\{$ kannan1, pramodv\}@illinois.edu
}

\begin{abstract}
The problem of designing near optimal strategies for multiple unicast traffic in wireline networks is wide-open; however, channel symmetry or traffic symmetry can be leveraged to show that routing can achieve with a poly-logarithmic approximation factor of the edge-cut bound. For the same problem, the edge-cut bound is known to only upper bound rates of routing flows and unlike the information theoretic cut-set bound, it does not upper bound (capacity-achieving) information rates with general strategies. In this paper, we demonstrate that under channel or traffic symmetry, the edge-cut bound upper-bounds general information rates, thus providing a capacity approximation result. The key technique is a combinatorial result relating edgecut bounds to generalized network sharing bounds.

Finally, we generalize the results to wireless networks via an intermediary class of combinatorial graphs known as polymatroidal networks - our main result is that a natural architecture separating the physical and networking layers is near optimal when the traffic is symmetric among source-destination pairs, even when the channel is asymmetric (due to asymmetric power constraints, or prior frequency allocation like frequency division duplexing). This result is complementary to our earlier work proving a similar result under channel symmetry [1].
\end{abstract}

\section{INTRODUCTION}

The central problem of network information theory is to characterize the capacity region of a general network. Wireline networks are a special class of such networks where the edges between vertices are unidirectional, orthogonal and noise-free. In this class of networks, network coding has the potential to provide significant advantages in comparison to flow (i.e. routing strategies) for multicast problems [2] as well as for multiple unicast problems [3]. Recent results due to Dougherty, Freiling, Zeger and Chan, Grant suggest that characterizing the capacity region of a multiple unicast network is a hard problem [4], [5], [6]. In particular, even coding strategies such as linear codes do not achieve capacity in general [5].

On the other hand, the literature on hardness of cut problems typically deal with edge-cut bounds which are conventional outer bounds on flow. But these bounds are not fundamental bounds on the capacity region [7], i.e. they can often be beaten if network coding is allowed. Although edge-cut bounds in directed networks are not fundamental, they are combinatorially well-represented. They are however, hard to approximate in general [8], [9].

One class of networks for which edge-cut bounds can be approximated well are undirected networks. Leighton and Rao show that for the problem of $k$-unicast in undirected networks, flow solutions approach the edge-cut bounds up to a factor of $\Theta(\log k)$ [10], [11]. There has also been discovered a semi-definite programming relaxation approach that allows an approximation of edge-cut bounds up to a factor of $\Theta(\sqrt{\log k})$ [12]. Interestingly, for undirected networks, edge-cut bounds can be derived from the vertex bipartition cutset bound and are hence, fundamental outer bounds on the capacity region. Thus, [10], [11] also characterize up to a factor of $\Theta(\log k)$ the capacity region of $k$-unicast in undirected networks. It has been conjectured that flow solutions in fact, achieve capacity [13], [14].

Another setting in which edge-cut bounds can be approximated well is the problem of multiple unicast in directed wireline networks with symmetric demands, i.e. for each source communicating to its destination at a certain rate, there is an independent message to be communicated from the destination back to the source at the same rate. Klein, Plotkin, Rao, Tardos [15] show under this model that flow solutions achieve within $\kappa \log ^{3}(k+1)$ of the edge-cut bounds. We ask the question: "Are these edge-cut bounds fundamental outer bounds on the capacity region?" Surprisingly, the answer turns out to be yes and the proof of this result is one of the main contributions of this paper. The key tool we use in the proof is the Generalized Network Sharing (GNS) bound that was first developed in [16] for directed wireline networks and was used subsequently also for two-unicast linear deterministic networks [17]. This completes an approximate characterization of the capacity region for this class of problems.

Additionally, consider the groupcast problem in directed wireline networks. There is a group of nodes and each node in the group has an independent message to be relayed to every other node in the group. [18] shows that the maximum sumrate achievable by routing flow for groupcast is at least half the multicut, a simple edge-cut based outer bound. We ask the question: "Is the multicut a fundamental outer bound on the sum-rate?". We show that the answer is no but that twice the

Parts of this paper were presented at the IEEE International Symposium on Information Theory 2012. 
multicut is indeed a fundamental outer bound. This shows that routing flow is approximately optimal for maximizing sum-rate in groupcast.

When there is some kind of symmetry in the network, either in the underlying graph (undirected or bidirected networks) or in the traffic (directed network with symmetric demands, sum-rate in groupcast), the following picture seems to emerge.

- (Achievability) Algorithmic Meta-Theorem: Edge-cut bounds can be well-approximated either by flows [10], [11], [15], [19], [18] or by other means [12].

- (Converse) Information-Theoretic Meta-Theorem: Edge-cut bounds are fundamental or close to fundamental outer bounds on the capacity region.

- Combined Meta-Theorem: Flows approximately achieve capacity.

In the second half of this paper, we extend these results to the wireless setting. The capacity region of multiple unicast in general wireless networks is an open problem in the general case. Recent work [20] [21] [1] has made progress in this direction by giving an approximate characterization of this capacity region by using the reciprocity in wireless channels, building on flow-cut gaps in undirected wireline networks. It has been shown that simple layered architectures involving local physical-layer schemes combined with global routing can achieve approximately optimal performance in wireless networks.

In many practical scenarios, the channel reciprocity may not hold due to asymmetric power constraints, directional antennas or frequency-duplexing. The question we address in this paper is: "do layered architectures continue to be near-optimal even in this case?" We answer this question in the affirmative under the symmetric demands model: there are $k$ specially-marked source-sink pairs of nodes $\left(s_{i}, t_{i}\right), i=1,2, \ldots, k$ with $s_{i}$ wanting to communicate an independent message to $t_{i}$ at rate $R_{i}$ and $t_{i}$ wanting to communicate an independent message to $s_{i}$ at rate $R_{i}$. This traffic model is relevant in several practical scenarios including voice calls, video calls, and interactive gaming.

Building on our results from wireline networks with symmetric demands, we show an analogous result for wireless networks with symmetric demands if it composed of channels, for which good schemes are known at a local physical layer level. Our results for wireless networks with symmetric demands include

1) Capacity approximations for networks comprised of Gaussian MAC and broadcast channels,

2) Degrees-of-freedom approximation for fixed Gaussian networks, and

3) Capacity approximations for fading Gaussian networks.

4) Capacity approximations for broadcast erasure networks with feedback.

At the heart of our achievable scheme is a connection to "polymatroidal networks" for which the symmetric demands problem was recently addressed, and it was shown that flow is within an inverse poly-logarithmic factor of the cut-set bound in [19]. The techniques for proving our achievable scheme closely mirror [1]. We need to derive our outer bound carefully; it is derived based on a suitable extension of the generalized network sharing bound [16] for wireless networks having a certain form. These results demonstrate the power of having the symmetry assumption in solving network capacity problems, which has been hitherto unexplored to the knowledge of the authors.

The rest of this paper is organized as follows. We set up notation and preliminaries in Section II. We then discuss

- $k$-unicast undirected networks in Section III,

- $k$-unicast directed symmetric-demand networks in Section IV,

- $k$-groupcast directed networks in Section V.

We then study a special class of Gaussian networks that we call MAC+BC networks in Section VI. We consider results for general Gaussian networks in Section VII. We study broadcast erasure networks with feedback in Section VIII. Finally, we conclude in Section IX.

\section{Preliminaries}

In this section, we will setup the wireline multiple-unicast problem with symmetric demands, along with the necessary notation.

Definition. A $k$-unicast wireline network $\mathcal{N}$ for source-destination pairs $\left\{\left(s_{i} ; d_{i}\right)\right\}_{i \in \mathcal{I}}$ with $|\mathcal{I}|=k$ (for instance, $\mathcal{I}:=$ $\{1,2, \ldots, k\})$ is a tuple $(\mathcal{G}, \underline{\mathrm{C}})$ where

- $\mathcal{G}=(\mathcal{V}, \mathcal{E})$ is the underlying directed or undirected graph with vertex set $\mathcal{V}$ and edge set $\mathcal{E}$, with $s_{i}, d_{i} \in \mathcal{V}(\mathcal{G})$ for $i \in \mathcal{I}$,

- $\underline{\mathrm{C}}=\left(C_{e}: e \in \mathcal{E}(\mathcal{G})\right)$ is the edge-capacity vector, with $C_{e} \in \mathbb{R}_{\geq 0} \cup\{\infty\} \forall e \in \mathcal{E}(\mathcal{G})$.

For each $i \in \mathcal{I}, s_{i}$ has independent information to be communicated to $d_{i}$ at rate $R_{i}$.

Notation. For directed graphs with $v \in \mathcal{V}(\mathcal{G})$, let $\ln (v)$ and $\operatorname{Out}(v)$ denote the edges entering into and leaving $v$ respectively. For undirected graphs with $v \in \mathcal{V}(\mathcal{G})$, we denote the set of edges incident on to $v$ as $\operatorname{lnc}(v)$.

Definition. Given a $k$-unicast network $\mathcal{N}=(\mathcal{G}, \underline{\mathrm{C}})$ for source-destination pairs $\left\{\left(s_{i} ; d_{i}\right)\right\}_{i \in \mathcal{I}}$, we say that the non-negative rate tuple $\left(R_{i}: i \in \mathcal{I}\right)$ is achievable if for any $\epsilon>0$, there exist positive integers $N$ and $T$ (called block length and number of epochs respectively), a finite alphabet $\mathcal{A}$ with $|\mathcal{A}| \geq 2$ and using notation $H_{v}:=\Pi_{i \in \mathcal{I}: v=s_{i}} \mathcal{A}^{\left\lceil N T R_{i}\right\rceil}$ (with an empty product being the singleton set), 
- for the case of directed graphs,

- encoding functions for $1 \leq t \leq T, e=(u, v) \in \mathcal{E}$, $f_{e, t}: H_{u} \times \Pi_{e^{\prime} \in \ln (u)}\left(\mathcal{A}^{\left\lfloor N C_{e^{\prime}}\right\rfloor}\right)^{(t-1)} \mapsto \mathcal{A}^{\left\lfloor N C_{e}\right\rfloor}$,

- decoding functions at destinations $d_{i}$ for $i \in \mathcal{I}$, $f_{d_{i}}: H_{d_{i}} \times \Pi_{e^{\prime} \in \ln \left(d_{i}\right)}\left(\mathcal{A}^{\left\lfloor N C_{e^{\prime}}\right\rfloor}\right)^{T} \mapsto \mathcal{A}^{\left\lceil N T R_{i}\right\rceil}$

- and for the case of undirected graphs,

- a subdivision of capacity for each edge $e=(u, v) \in \mathcal{E}$ and each epoch $t, 1 \leq t \leq T, C_{e, t}^{u}, C_{e, t}^{v} \geq 0$ such that $C_{e, t}^{u}+C_{e, t}^{v} \leq C_{e}$, where $C_{e, t}^{u}$ is the capacity for outgoing data from $u$ on edge $e$,

- for $1 \leq t \leq T, e=(u, v) \in \mathcal{E}$, two encoding functions $f_{e, t}^{u}, f_{e, t}^{v}$ as $f_{e, t}^{u}: H_{u} \times \Pi_{e^{\prime}=(u, w) \in \operatorname{lnc}(u)} \Pi_{l=1}^{t-1} \mathcal{A}^{\left\lfloor N C_{e^{\prime}, l}^{w}\right\rfloor} \mapsto \mathcal{A}^{\left\lfloor N C_{e, t}^{u}\right\rfloor}$, $f_{e, t}^{v}: H_{v} \times \Pi_{e^{\prime}=(v, w) \in \operatorname{lnc}(v)} \Pi_{l=1}^{t-1} \mathcal{A}^{\left\lfloor N C_{e^{\prime}, l}^{w}\right\rfloor} \mapsto \mathcal{A}^{\left\lfloor N C_{e, t}^{v}\right\rfloor}$,

- decoding functions at destinations $d_{i}$ for $i \in \mathcal{I}$, $f_{d_{i}}: H_{d_{i}} \times \Pi_{e^{\prime}=\left(d_{i}, w\right) \in \operatorname{lnc}\left(d_{i}\right)} \Pi_{l=1}^{T} \mathcal{A}^{\left\lfloor N C_{e^{\prime}, l}^{w}\right\rfloor} \mapsto \mathcal{A}^{\left\lceil N T R_{i}\right\rceil}$

with the property that under the uniform probability distribution on $\Pi_{i \in \mathcal{I}} \mathcal{A}^{\left\lceil N T R_{i}\right\rceil}$,

$$
\operatorname{Pr}\left(g\left(m_{1}, m_{2}, \ldots, m_{k}\right) \neq\left(m_{1}, m_{2}, \ldots, m_{k}\right)\right) \leq \epsilon,
$$

where $g: \Pi_{i \in \mathcal{I}} \mathcal{A}^{\left\lceil N T R_{i}\right\rceil} \mapsto \Pi_{i \in \mathcal{I}} \mathcal{A}^{\left\lceil N T R_{i}\right\rceil}$ is the global decoding function induced inductively by

- $\left\{f_{e, t}: e \in \mathcal{E}(\mathcal{G}), 1 \leq t \leq T\right\}$ and $\left\{f_{d_{i}}: i \in \mathcal{I}\right\}$ in the directed graph case and

- $\left\{f_{e, t}^{u}, f_{e, t}^{v}: e=(u, v) \in \mathcal{E}(\mathcal{G}), 1 \leq t \leq T\right\}$ and $\left\{f_{d_{i}}: i \in \mathcal{I}\right\}$ in the undirected graph case.

The closure of the set of achievable rate tuples is called the capacity region and is denoted by $\mathcal{C}$. Define the sum-rate-capacity by $C_{\text {sum-rate }}:=\sup _{\left(R_{i}: i \in \mathcal{I}\right) \in \mathcal{C}} \sum_{i \in \mathcal{I}} R_{i}$.

Definition. Given a $k$-unicast network $\mathcal{N}=(\mathcal{G}, \underline{\mathrm{C}})$ for source-destination pairs $\left\{\left(s_{i} ; d_{i}\right)\right\}_{i \in \mathcal{I}}$, we say that the non-negative rate tuple $\left(R_{i}: i \in \mathcal{I}\right)$ is achievable by routing flow if there exist for each $i \in \mathcal{I}$ and each $e=(u, v) \in \mathcal{E}(\mathcal{G})$, real numbers $f_{i, e} \geq 0$ in the directed graph case and $f_{i, e}^{u}, f_{i, e}^{v} \geq 0$ in the undirected graph case such that

- $\sum_{i \in \mathcal{I}} f_{i, e} \leq C_{e} \forall e \in \mathcal{E}(\mathcal{G})$, and for each $i \in \mathcal{I}$ and each $v \in \mathcal{V}(\mathcal{G})$,

$$
\sum_{e \in \operatorname{Out}(v)} f_{i, e}-\sum_{e \in \ln (v)} f_{i, e}= \begin{cases}0 & \text { if } v \neq s_{i}, d_{i}, \\ R_{i} & \text { if } v=s_{i}, \\ -R_{i} & \text { if } v=d_{i} .\end{cases}
$$

in the directed graph case and

- $\sum_{i \in \mathcal{I}} f_{i, e}^{u}+f_{i, e}^{v} \leq C_{e} \forall e=(u, v) \in \mathcal{E}(\mathcal{G})$, and for each $i \in \mathcal{I}$ and each $v \in \mathcal{V}(\mathcal{G})$,

$$
\sum_{e=(v, w) \in \operatorname{lnc}(v)} f_{i, e}^{v}-f_{i, e}^{w}= \begin{cases}0 & \text { if } v \neq s_{i}, d_{i}, \\ R_{i} & \text { if } v=s_{i}, \\ -R_{i} & \text { if } v=d_{i} .\end{cases}
$$

in the undirected graph case.

The closure of the set of rate tuples achievable by routing flow is called the flow region and is denoted by $\mathcal{F}$. Define the sum-rate-max-flow by $F_{\text {sum-rate }}:=\sup _{\left(R_{i}: i \in \mathcal{I}\right) \in \mathcal{F}} \sum_{i \in \mathcal{I}} R_{i}$.

Definition. Given a $k$ unicast network $\mathcal{N}=(\mathcal{G}, \underline{\mathrm{C}})$ for source-destination pairs $\left\{\left(s_{i} ; d_{i}\right)\right\}_{i \in \mathcal{I}}$, we define the edge-cut outer bound denoted by $\mathcal{R}_{\text {edge-cut }}$, to be the set of all non-negative tuples $\left(R_{i}: i \in \mathcal{I}\right)$ that satisfy for every $E \subseteq \mathcal{E}(\mathcal{G})$, the inequality $\sum_{i \in J} R_{i} \leq \sum_{e \in E} C_{e}$ where index $i \in J \subseteq \mathcal{I}$ if and only if $\mathcal{G} \backslash E$ has no paths from $s_{i}$ to $d_{i}$. (The paths are directed paths in the directed graph case and undirected paths in the undirected graph case.) We define the multicut denoted by $R_{\text {multicut }}$, to be the minimum value of $\sum_{e \in E} C_{e}$ over all $E \subseteq \mathcal{E}(\mathcal{G})$ with the property that $\mathcal{G} \backslash E$ has no paths from $s_{i}$ to $d_{i}$ for each $i \in \mathcal{I}$.

While it is clear that $\mathcal{F} \subseteq \mathcal{R}_{\text {edge-cut }}$ and $\mathcal{F} \subseteq \mathcal{C}$, the connection between $\mathcal{C}$ and $\mathcal{R}_{\text {edge-cut }}$ is unclear. It is easy to show

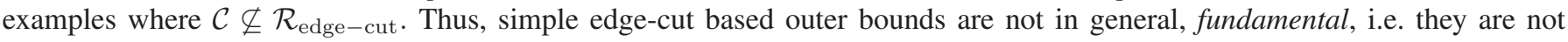
outer bounds on the capacity region. Likewise it is clear that $F_{\text {sum-rate }} \leq R_{\text {multicut }}$ and $F_{\text {sum-rate }} \leq C_{\text {sum-rate }}$ but $C_{\text {sum-rate }}$ and $R_{\text {multicut }}$ have no apparent connection. Indeed, [3] provides a series of $k$-unicast networks, one for each $k$ with $k=2^{n}$ with $F_{\text {sum-rate }}=R_{\text {multicut }}=\frac{1}{k} C_{\text {sum-rate }}$ and $\mathcal{C} \nsubseteq(k-\epsilon) \mathcal{R}_{\text {edge-cut }}$ for any $\epsilon>0$.

Remark 1. Note that $R_{\text {multicut }}$ may in general be strictly larger than the tighter bound on $F_{\text {sum-rate }}$ given by $\sup _{\left(R_{i}: i \in \mathcal{I}\right) \in \mathcal{R}_{\text {edge-cut }}} \sum_{i \in \mathcal{I}} R_{i}$ 


\section{III. $k$-UNICAST UNDIRECTED NETWORKS}

Theorems 1, 2 and Corollary 3 will refer to $k$-unicast undirected networks.

Theorem 1. (Leighton-Rao [10], Linial-London-Rabinovich [11])

$$
\frac{\mathcal{R}_{\text {edge-cut }}}{\Theta(\log k)} \subseteq \mathcal{F} \subseteq \mathcal{R}_{\text {edge-cut }}
$$

Theorem 2.

$$
\mathcal{C} \subseteq \mathcal{R}_{\text {edge-cut }}
$$

Theorems 1 and 2 together imply that routing flow is approximately capacity-achieving:

Corollary 3.

$$
\frac{\mathcal{R}_{\text {edge-cut }}}{\Theta(\log k)} \subseteq \mathcal{F} \subseteq \mathcal{C} \subseteq \mathcal{R}_{\text {edge-cut }}
$$

Although Theorem 2 is easy to show, it is not very well-known. So, we provide a proof here:

Proof: Consider a $k$-unicast undirected network $\mathcal{N}$ for source-destination pairs $\left\{\left(s_{i} ; d_{i}\right)\right\}_{i \in \mathcal{I}}$. Consider a coding scheme that achieves rates $\left(R_{i}: i \in \mathcal{I}\right)$ in $T$ epochs of block length $N$ with overall error probability at most $\epsilon$. Let $E \subseteq \mathcal{E}(\mathcal{G})$ be any subset of edges and let $J \subseteq \mathcal{I}$ denote the set of all indices $i \in \mathcal{I}$ which have $s_{i}, d_{i}$ disconnected from each other in $\mathcal{G} \backslash E$. Let $\left(\mathcal{V}_{1}, \mathcal{V}_{2}, \ldots, \mathcal{V}_{r}\right)$ denote the partition of $\mathcal{V}$ obtained as the connected components of $\mathcal{G} \backslash E$ and let $E^{\prime} \subseteq E$ denote the set of edges that connect a vertex in $\mathcal{V}_{s}$ to a vertex in $\mathcal{V}_{s^{\prime}}$, for $1 \leq s, s^{\prime} \leq r, s \neq s^{\prime}$.

For $1 \leq q \leq r$, let $J_{q}$ denote the set of all indices $i \in \mathcal{I}$ which have $s_{i} \in \mathcal{V}_{q}$ and $d_{i} \notin \mathcal{V}_{q}$. $\left(J_{1}, J_{2}, \ldots, J_{r}\right)$ is a partition of $J$. For $1 \leq q \leq r$, let $X_{q}$ denote the vector of all symbols transmitted on edges going out of $\mathcal{V}_{q}$ and in to $\mathcal{V}_{s}$ for some $s \neq q$. For $i \in \mathcal{I}$, let $W_{i}$ be the source message at $s_{i}$ that is required to be decoded at $d_{i}$. By the cutset bound written for vertex bipartition $\left(\mathcal{V}_{q}, \mathcal{V} \backslash \mathcal{V}_{q}\right)$, we have

$$
\sum_{i \in J_{q}} H\left(W_{i}\right) \leq H\left(X_{q}\right)
$$

Adding up these inequalities over $1 \leq q \leq r$, we get $\sum_{i \in J} H\left(W_{i}\right) \leq \sum_{q=1}^{r} H\left(X_{q}\right)$ which yields $\sum_{i \in J} R_{i} \leq \sum_{e \in E^{\prime}} C_{e} \leq$ $\sum_{e \in E} C_{e}$. This establishes $\mathcal{C} \subseteq \mathcal{R}_{\text {edge-cut }}$.

Remark 2. Theorems analogous to 1 and 2 can be similarly proved for $k$-unicast in bidirected networks, i.e. directed networks in which for every edge from node $u$ to node $v$, there is another edge from node $v$ to node $u$ with the same capacity.

\section{IV. $k$-PAIR UNICAST DIRECTED SYMMETRIC-DEMAND NETWORKS}

Definition. A $k$-pair unicast directed symmetric-demand network is a $2 k$-unicast directed network $\mathcal{N}$ with $2 k$ distinct distinguished nodes (source-destination nodes) $u_{1}, u_{2}, \ldots, u_{k}, v_{1}, v_{2}, \ldots, v_{k}$ with source-destination pairs $\left\{s_{i} ; d_{i}\right\}_{i \in \mathcal{I}}$ where $\mathcal{I}=$ $\{1,2, \ldots, k\} \cup\{-1,-2, \ldots,-k\}$ and for $i>0, s_{i}=u_{i}, d_{i}=v_{i}$, while for $i<0, s_{i}=v_{-i}, d_{i}=u_{-i}$. The rate tuple $\left(R_{i}: 1 \leq i \leq k\right)$ is defined to be in the capacity region $\mathcal{C}$, flow region $\mathcal{F}$, edge-cut outer bound $\mathcal{R}_{\text {edge-cut }}$ for the $k$-pair unicast directed symmetric-demand network if the rate tuple $\left(R_{i}^{\prime}: i \in \mathcal{I}\right)$, given by $R_{i}^{\prime}=R_{|i|}$ for $i \in \mathcal{I}$, lies in the capacity region, flow region, edge-cut outer bound respectively of the $2 k$-unicast directed network.

Remark 3. There is no loss of generality in assuming $u_{1}, u_{2}, \ldots, u_{k}, v_{1}, v_{2}, \ldots, v_{k}$ distinct since if they aren't, we can add more nodes and infinite capacity edges to make them distinct while obtaining a network with identical capacity region.

Definition. Given a directed network $\mathcal{N}=(\mathcal{G}, \underline{\mathrm{C}})$ with a set of $2 r$ distinct distinguished vertices $w_{1}, w_{2}, \ldots, w_{r}, w_{1}^{\prime}, w_{2}^{\prime}, \ldots, w_{r}^{\prime}$, with $w_{i}$ communicating to $w_{i}^{\prime}$ at rate $R_{i}$, and possibly other sources and destinations with their independent messages. If a set of edges $E \subseteq \mathcal{E}(\mathcal{G})$ has the property that $\mathcal{G} \backslash E$ has no directed paths from $w_{i}$ to $w_{j}^{\prime}$ whenever $\pi(i) \geq \pi(j), 1 \leq i, j \leq r$, for some permutation $\pi:\{1,2, \ldots, r\} \mapsto\{1,2, \ldots, r\}$, then we say that $E$ is a $G N S$-cut for $\left\{w_{1}, w_{2}, \ldots, w_{r} ; w_{1}^{\prime}, w_{2}^{\prime}, \ldots, w_{r}^{\prime}\right\}$ with permutation $\pi$.

Definition. Given a $k$-pair unicast directed symmetric-demand network $\mathcal{N}=(\mathcal{G}, \underline{\mathrm{C}})$ with source-destination nodes $u_{1}, u_{2}, \ldots, u_{k}, v_{1}, v_{2}, \ldots$, we define the GNS-cut outer bound denoted by $\mathcal{R}_{\mathrm{GNS}-\text { cut }}$, to be the set of all non-negative tuples $\left(R_{i}: 1 \leq i \leq k\right)$ that satisfy for every $E \subseteq \mathcal{E}(\mathcal{G})$, the inequality $\sum_{i \in J} R_{i} \leq \sum_{e \in E} C_{e}$ whenever $E$ is a GNS-cut for $\left\{w_{1}, w_{2}, \ldots, w_{r} ; w_{1}^{\prime}, w_{2}^{\prime}, \ldots, w_{r}^{\prime}\right\}$ with some permutation $\pi$ where

- $J \subseteq\{1,2, \ldots, k\},|J|=r$,

- $w_{1}, w_{2}, \ldots, w_{r}, w_{1}^{\prime}, w_{2}^{\prime}, \ldots, w_{r}^{\prime}$ are distinct,

- for $1 \leq j \leq r,\left(w_{j}, w_{j}^{\prime}\right)=\left(u_{i}, v_{i}\right)$ or $\left(v_{i}, u_{i}\right)$ for some $i \in J$.

We define a weak edge-cut outer bound for this class of networks. 
Definition. Given a $k$-pair unicast directed symmetric-demand network $\mathcal{N}=(\mathcal{G}, \underline{\mathrm{C}})$ with source-destination nodes $u_{1}, u_{2}, \ldots, u_{k}, v_{1}, v_{2}, \ldots$ we define the weak edge-cut outer bound denoted by $\mathcal{R}_{\text {w.e.c. }}$, to be the set of all non-negative tuples $\left(R_{i}: 1 \leq i \leq k\right)$ that satisfy for every $E \subseteq \mathcal{E}(\mathcal{G})$, the inequality $\sum_{i \in J} R_{i} \leq \sum_{e \in E} C_{e}$ where index $i \in J \subseteq\{1,2, \ldots, k\}$ if and only if $\mathcal{G} \backslash E$ has no directed paths from either $u_{i}$ to $v_{i}$ or $v_{i}$ to $u_{i}$ or both.

Remark 4. For $E \subseteq \mathcal{E}(\mathcal{G})$, if $J_{1}$ is the set of indices $i, 1 \leq i \leq k$ for which $\mathcal{G} \backslash E$ has no directed paths from either $u_{i}$ to $v_{i}$ or from $v_{i}$ to $u_{i}$ but not both and $J_{2}$ is the set of indices $i, 1 \leq i \leq k$ for which $\mathcal{G} \backslash E$ has no directed paths from $u_{i}$ to $v_{i}$ and from $v_{i}$ to $u_{i}$, then the edge-cut outer bound has the inequality $\sum_{i \in J_{1}} R_{i}+2 \sum_{j \in J_{2}} R_{j} \leq \sum_{e \in E} C_{e}$ while the weak edge-cut outer bound has the inequality $\sum_{i \in J_{1}} R_{i}+\sum_{j \in J_{2}} R_{j} \leq \sum_{e \in E} C_{e}$. It is therefore, clear that

$$
\mathcal{R}_{\text {edge-cut }} \subseteq \mathcal{R}_{\text {w.e.c. }} \subseteq 2 \mathcal{R}_{\text {edge-cut }} \text {. }
$$

Theorems 4, 5 and 6 and Corollary 7 will refer to $k$-pair unicast directed symmetric-demand networks.

Theorem 4. (Klein-Plotkin-Rao-Tardos [15])

$$
\frac{\mathcal{R}_{\text {w.e.c. }}}{\kappa\left(\log ^{3}(k+1)\right)} \subseteq \mathcal{F} \subseteq \mathcal{R}_{\text {w.e.c. }}
$$

for a universal constant $\kappa$.

\section{Theorem 5.}

$$
\mathcal{C} \subseteq \mathcal{R}_{\mathrm{GNS}-\text { cut }}
$$

Theorem 6.

$$
\mathcal{R}_{\text {w.e.c. }}=\mathcal{R}_{\mathrm{GNS}-\text { cut }}
$$

Theorems 4, 5 and 6 together imply that routing flow is approximately capacity-achieving:

\section{Corollary 7.}

$$
\frac{\mathcal{R}_{\text {w.e.c. }}}{\kappa\left(\log ^{3}(k+1)\right)} \subseteq \mathcal{F} \subseteq \mathcal{C} \subseteq \mathcal{R}_{\text {w.e.c. }}=\mathcal{R}_{\mathrm{GNS}-\text { cut }},
$$

for a universal constant $\kappa$.

Remark 5. The GNS bound is to the capacity region what the edge-cut bound is to the flow region, namely an intuitive outer bound that arises from simple connectivity properties of the underlying graph of the network. While more sophisticated bounds [7], [3], [22] include the GNS bound as a special case, it is the simplicity of the GNS bound that becomes useful for Theorem 6, which is proved by showing that weak edge-cuts and GNS-cuts are identical for k-pair directed symmetric-demand networks. We also note that the outer bound $\mathcal{R}_{\mathrm{GNS}-\mathrm{cut}}$ is in general, strictly tighter than the cutset outer bound and that in general, the capacity region $\mathcal{C}$ is not contained in $\mathcal{R}_{\text {edge-cut }}$ although it is always contained in $\mathrm{R}_{\mathrm{w} . e . c .}$.

Conjecture 8. (Analog of Li and Li conjecture [13], [14]) For k-pair unicast directed symmetric-demand networks,

$$
\mathcal{F} \subseteq \mathcal{C} \subseteq 2 \mathcal{F}
$$

i.e. network coding can improve rates beyond routing flow by at most a factor 2.

This conjecture can be considered a stronger version of the $\mathrm{Li}$ and $\mathrm{Li}$ conjecture inasmuch as the capacity region of an arbitrary $k$-unicast bidirected or undirected network can be characterized up to a factor 2 , if the capacity region of a $k$-unicast directed symmetric-demand network can be characterized.

We first present the proof of Theorem 5. The essential idea is contained in [16] but we provide a proof here for completeness.

Proof: Consider a communication scheme over alphabet $\mathcal{A}$ with block length $N$ and number of epochs $T$ that achieves for $1 \leq i \leq r$, rate $R_{i}$ for the message from $w_{i}$ to $w_{i}^{\prime}$ with error probability at most $\epsilon$. Let $E$ be a GNS-cut for $\left\{w_{1}, w_{2}, \ldots, w_{r} ; w_{1}^{\prime}, w_{2}^{\prime}, \ldots, w_{r}^{\prime}\right\}$ which may assumed to be with the identity permutation without loss of generality. For $1 \leq i \leq r$, let $W_{i}$ be the source message at $w_{i}$ that is required to be delivered to $w_{i}^{\prime}$. Let $W_{0}$ denote the vector of all other source messages in the network. $W_{0}, W_{1}, \ldots, W_{r}$ are mutually independent and each $W_{i}, 0 \leq i \leq r$ has the uniform distribution. Let $X_{E}$ denote the vector of all symbols transmitted on the edges of $E$ over the duration of the complete scheme. For $1 \leq i \leq r$, let $\hat{W}_{i}$ denote the estimate at $w_{i}^{\prime}$ of the source message $W_{i}$ upon completion of the coding scheme. Assume $|\mathcal{A}|=2$; the proof for larger alphabet size 
is identical. Note that

$$
\begin{aligned}
H & \left(W_{1}, W_{2}, \ldots, W_{r} \mid X_{E}, W_{0}\right) \\
= & \sum_{i=1}^{r} H\left(W_{i} \mid X_{E}, W_{0},\left\{W_{j}: 1 \leq j<i\right\}\right) \\
= & \sum_{i=1}^{r} H\left(W_{i} \mid X_{E}, W_{0},\left\{W_{j}: 1 \leq j<i\right\}, \hat{W}_{i}\right) \\
& {\left[\text { since } \hat{W}_{i} \text { is a function of } X_{E}, W_{0},\left\{W_{j}: 1 \leq j<i\right\}\right.} \\
& \quad \text { from the connectivity properties of } \mathcal{G} \backslash E] \\
\leq & \sum_{i=1}^{r} H\left(W_{i} \mid \hat{W}_{i}\right) \\
\leq & \sum_{i=1}^{r} h(\epsilon)+\epsilon\left\lceil N T R_{i}\right\rceil \leq r h(\epsilon)+\epsilon \sum_{i=1}^{r}\left\lceil N T R_{i}\right\rceil,
\end{aligned}
$$

where $h(\cdot)$ is the binary entropy function. Thus, we have

$$
\begin{aligned}
& \sum_{i=1}^{r}\left\lceil N T R_{i}\right\rceil=H\left(W_{1}, W_{2}, \ldots, W_{r}\right) \\
& \begin{array}{r}
=I\left(W_{1}, W_{2}, \ldots, W_{r} ; X_{E}, W_{0}\right) \\
\quad+H\left(W_{1}, W_{2}, \ldots, W_{r} \mid X_{E}, W_{0}\right)
\end{array} \\
& \leq I\left(W_{1}, W_{2}, \ldots, W_{r} ; X_{E} \mid W_{0}\right)+r h(\epsilon)+\epsilon \sum_{i=1}^{r}\left\lceil N T R_{i}\right\rceil \\
& \leq H\left(X_{E}\right)+r h(\epsilon)+\epsilon \sum_{i=1}^{r}\left\lceil N T R_{i}\right\rceil \\
& \leq \sum_{e \in E} T\left\lfloor N C_{e}\right\rfloor+r h(\epsilon)+\epsilon \sum_{i=1}^{r}\left\lceil N T R_{i}\right\rceil
\end{aligned}
$$

This establishes that $\sum_{i=1}^{r} R_{i} \leq \sum_{e \in E} C_{e}$. Hence, we have $\mathcal{C} \subseteq \mathcal{R}_{\mathrm{GNS}-\text { cut }}$.

Now, we prove the equivalence between weak edge-cuts and GNS-cuts for $k$-pair unicast directed symmetric-demand networks, thus proving Theorem 6.

Proof: It is easy to see that the inequality obtained from a GNS-cut can always be obtained from a weak edge-cut since a GNS-cut requires stronger disconnections as compared to a weak edge-cut. This gives $\mathcal{R}_{\text {w.e.c. }} \subseteq \mathcal{R}_{\text {GNS-cut }}$. To show $\mathcal{R}_{\text {GNS-cut }} \subseteq \mathcal{R}_{\text {w.e.c. }}$, we now consider $E \subseteq \mathcal{E}(\mathcal{G})$, and say $i \in J \subseteq\{1,2, \ldots, k\}$ if and only if $\mathcal{G} \backslash E$ has no directed paths from either $u_{i}$ to $v_{i}$ or from $v_{i}$ to $u_{i}$ or both. We show that $E$ is a GNS-cut for $\left\{w_{1}, w_{2}, \ldots, w_{r} ; w_{1}^{\prime}, w_{2}^{\prime}, \ldots, w_{r}^{\prime}\right\}$ with some permutation $\pi$ where the $2 r$ vertices $w_{1}, w_{2}, \ldots, w_{r}, w_{1}^{\prime}, w_{2}^{\prime}, \ldots, w_{r}^{\prime}$ are all distinct and for $1 \leq j \leq r,\left(w_{j}, w_{j}^{\prime}\right)=\left(u_{i}, v_{i}\right)$ or $\left(v_{i}, u_{i}\right)$ for some $i \in J$ with $|J|=r$. We will prove this for the case $J=\{1,2, \ldots, k\}$. The proof for other choices of $J$ is similar.

Define the connectivity graph $\mathcal{G}_{c}$ as a directed graph over $2 k$ vertices $u_{1}, u_{2}, \ldots, u_{k}, v_{1}, v_{2}, \ldots, v_{k}$ as follows. For every pair of distinct vertices $w$ and $z$, there is a directed edge from $w$ to $z$ in $\mathcal{G}_{c}$ if and only if $w$ has a directed path to $z$ in $\mathcal{G} \backslash E$. See Fig. 1(a) for an example. $\mathcal{G}_{c}$ is transitively closed, i.e. for three distinct vertices $w, z, x$, if $w$ has an edge to $z$ and $z$ has an edge to $x$, then $w$ has an edge to $x$. Define two distinct vertices $u$ and $v$ in $\mathcal{G}_{c}$ as associated, if $u$ has an edge to $v$ and $v$ has an edge to $u$. If we define every vertex to be associated with itself, this relation is reflexive and symmetric. As $\mathcal{G}_{c}$ is transitively closed, this relation is also transitive and so, association is an equivalence relation. Further, for each $i=1,2, \ldots, k$, we have that $u_{i}$ and $v_{i}$ are not associated since there are no paths in $\mathcal{G} \backslash S$ from either $u_{i}$ to $v_{i}$ or from $v_{i}$ to $u_{i}$.

Now, define the reduced connectivity graph $\mathcal{G}_{r}$ as a directed graph with vertices represented by the equivalence classes defined from being associated in $\mathcal{G}_{c}$. See Fig. 1(b) for an example. There is a directed edge from equivalence class $\mathcal{E}_{1}$ to $\mathcal{E}_{2}$ in $\mathcal{G}_{r}$ if there is a directed edge in $\mathcal{G}_{c}$ from each vertex in $\mathcal{E}_{1}$ to each vertex in $\mathcal{E}_{2}$. By transitive closure of $\mathcal{G}_{c}$, this happens if and only if there is a directed edge in $\mathcal{G}_{c}$ from some vertex in $\mathcal{E}_{1}$ to some vertex in $\mathcal{E}_{2}$. $\mathcal{G}_{r}$ has at least two vertices since $u_{1}$ and $v_{1}$ cannot belong to the same equivalence class.

Now, note that $\mathcal{G}_{r}$ is a directed acyclic graph. Suppose not, i.e. suppose the equivalence classes $\mathcal{E}_{1}, \mathcal{E}_{2}, \mathcal{E}_{3}, \ldots, \mathcal{E}_{r}, \mathcal{E}_{1}$ in that order describe a directed cycle. Then, in the graph $\mathcal{G}_{c}$, for vertex $w_{j}$ chosen from equivalence class $\mathcal{E}_{j}$ for $j=1,2, \ldots, r$, we have $w_{j}$ has a directed edge to $w_{j+1}$ for $j=1,2, \ldots, r-1$ and $w_{r}$ has a directed edge to $w_{1}$. Transitive closure of $\mathcal{G}_{c}$ implies 


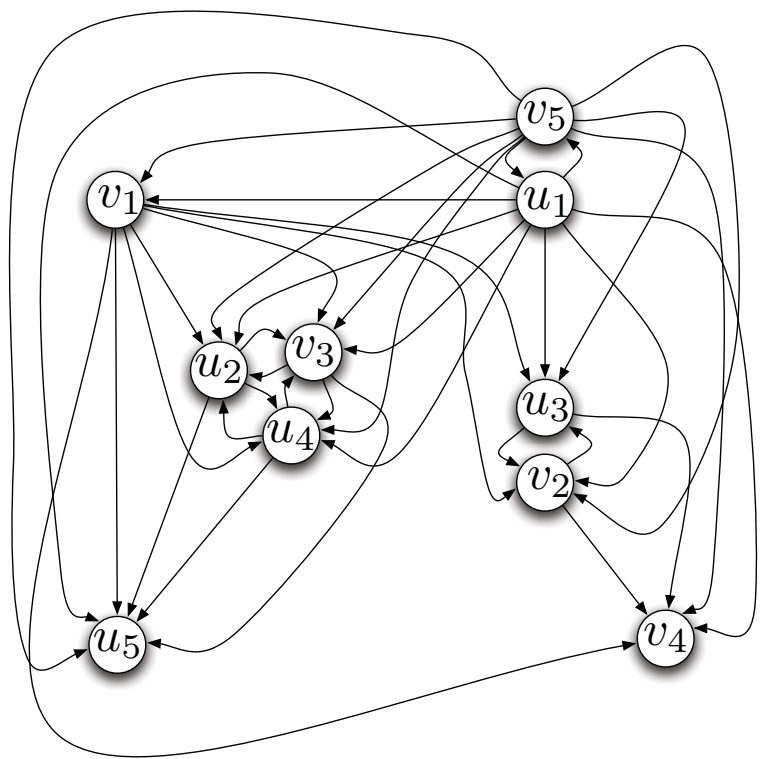

(a) Connectivity graph $\mathcal{G}_{c}$

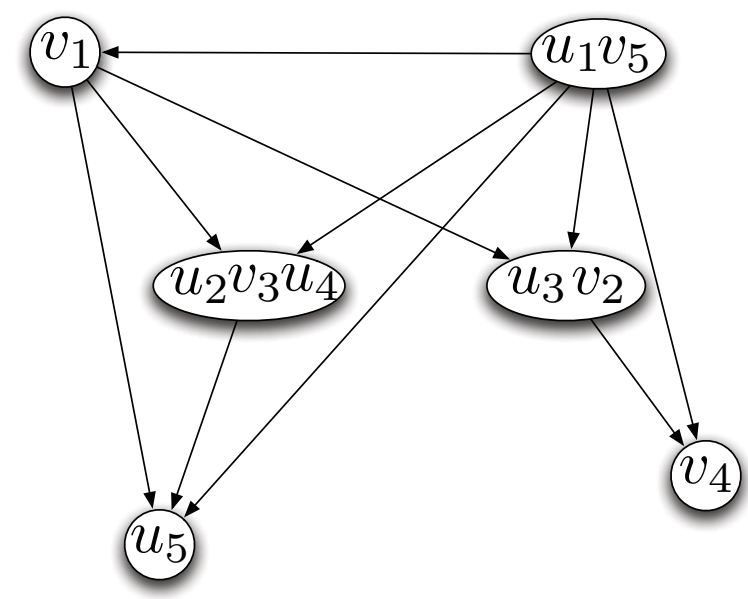

(b) Reduced connectivity graph $\mathcal{G}_{r}$

Fig. 1. Connectivity graph and reduced connectivity graph for an instance of a 5-pair unicast directed symmetric-demand network

that there must be a directed edge from $w_{j}$ to $w_{k}$ for $j, k=1,2, \ldots, r, j \neq k$, leading to a collapse of the $r \geq 2$ equivalence classes into one equivalence class, a contradiction.

We now describe an algorithm $\mathcal{P}$ that fills the cells of a $k \times 2$ table with vertex names from $u_{1}, u_{2}, \ldots, u_{k}, v_{1}, v_{2}, \ldots, v_{k}$ such that the following properties hold:

$(\alpha)$ Each vertex shows up exactly once in the table.

( $\beta$ ) Each row of the table is made up of vertices $u_{i}$ and $v_{i}$ for some $i$.

$(\gamma)$ In graph $\mathcal{G}_{c}$, vertex $u$ obtained from the first column of row $i$ does not have an edge to vertex $v$ obtained from the second column of row $j$ whenever $i \geq j$.

A directed acyclic graph has at least one sink vertex, i.e. a vertex with no outgoing edges. This is the proposed algorithm $\mathcal{P}$.

(1) Pick any sink vertex in directed acyclic graph $\mathcal{G}_{r}$.

(2) List the vertices of $\mathcal{G}_{c}$ in the equivalence class represented by the chosen sink vertex.

(a) Pick a vertex $w$ from the list.

(b) If vertex $w$ has been entered previously in the table, do nothing. Else, add vertex $w$ in the first column of the lowest row in the table not yet filled. Add the destination of vertex $w$ in the second column of the same row, i.e. if $v_{3}$ was entered in the first column of the lowest available row, then fill $u_{3}$ in the second column.

(c) Remove $w$ from the list and go back to (a) if the list is still non-empty, else proceed to (3)

(3) Modify graph $\mathcal{G}_{r}$ by deleting the chosen sink vertex. The modified graph continues to be a directed acyclic graph. If this graph has non-zero number of vertices, go to step (1), else quit.

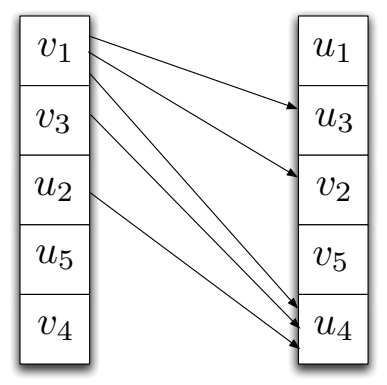

Fig. 2. One of the several $5 \times 2$ tables generated by running algorithm $\mathcal{P}$ on the $\mathcal{G}_{c}, \mathcal{G}_{r}$ shown in Fig. 1 . The order of choosing sinks was $v_{4}, u_{5}, u_{2} v_{3} u_{4}$, $u_{3} v_{2}, v_{1}, u_{1} v_{5}$. The arrows show connectivity from the vertices on the left to the vertices on the right in $\mathcal{G}_{c}$. Note that no arrows go 'horizontally' or go 'upward'. They always go 'downward' which is the desired GNS-cut property.

Let us verify the claimed properties. By step (b), it is clear that each non-empty row of the table is filled with a vertex http://mc.manuscriptcentral.com/t-it 
and its destination, i.e. the vertices $u_{i}$ and $v_{i}$ for some $i$. As the algorithm terminates only when all vertices have been listed and checked for their presence in the table, and as the vertices are added only when they have not been added previously, it follows that each vertex shows up exactly once and the table is completely filled upon termination of the algorithm. This verifies claimed properties $(\alpha)$ and $(\beta)$. Now, we verify property $(\gamma)$.

- Consider vertices in row $j$ of the table, say $w$ and $w^{\prime}$ with $w$ in the first column. These are source-destination pairs $u_{i}, v_{i}$ for some $i$. We claim that $w$ has no edge to $w^{\prime}$ in $\mathcal{G}_{c}$. Suppose it did. Then, there would be an edge in $\mathcal{G}_{r}$ from the equivalence class $\mathcal{E}$ containing $w$ to the equivalence class $\mathcal{E}^{\prime}$ containing $w^{\prime}$. These equivalence classes must be distinct as $w$ and $w^{\prime}$ are source-destination pairs. This means that the algorithm $\mathcal{P}$ must pick the equivalence class containing $w^{\prime}$ before picking the equivalence class containing $w$. When $w^{\prime}$ is probed in the list of vertices, $w$ must not have been entered in to the table as yet, and thus $w^{\prime}$ would then be entered in the first column of some row and $w$ in the second column of the same row. This contradicts the assumed structure of the table. Thus, we have no edge from $w$ to $w^{\prime}$ in $\mathcal{G}_{c}$.

- Now, consider rows $i$ and $j$ with $i>j$. Let the vertices in row $i$ be $w$ and $w^{\prime}$ with $w$ in the first column and the vertices in row $j$ be $z$ and $z^{\prime}$ with $z$ in the first column. We claim that there is no edge from $w$ to $z^{\prime}$ in $\mathcal{G}_{c}$. Suppose there is. Then, either $w$ and $z^{\prime}$ are in the same equivalence class in $\mathcal{G}_{c}$ or they are not. If they are not, then the equivalence class containing $z^{\prime}$ has an incoming edge from the equivalence class containing $w$ and thus, the former ought to have been picked by the algorithm before the latter. This is inconsistent with the table which was filled with $w$ in the first column of a row while $z^{\prime}$ had not yet been filled. Now, if $w$ and $z^{\prime}$ are in the same equivalence class, then clearly $z$ does not fall in that equivalence class. Moreover, the equivalence class containing $z$ is picked after the equivalence class containing $w$ and $z^{\prime}$. The algorithm $\mathcal{P}$, when exhausting the list of vertices in the equivalence class containing $w$ and $z^{\prime}$ is supposed to have accepted $w$ and added it to the first column of a row and rejected $z^{\prime}$. But when $z^{\prime}$ was probed, we are still in the same equivalence class as $w$, so $z$ had not been probed yet. Then $z^{\prime}$ must have been added to the first column of some row, which contradicts the structure of the table. Thus, there is no edge from $w$ to $z^{\prime}$ in $\mathcal{G}_{c}$.

Now, if the $j^{\text {th }}$ row of the table consists of $u_{i}, v_{i}$, we set $\pi(j)=i$ and $\left(w_{i}, w_{i}^{\prime}\right)=\left(u_{i}, v_{i}\right)$ or $\left(v_{i}, u_{i}\right)$ depending on whether the first entry in the row is $u_{i}$ or $v_{i}$. This shows that $S$ is a GNS-cut for $\left\{w_{1}, w_{2}, \ldots, w_{k} ; w_{1}^{\prime}, w_{2}^{\prime}, \ldots, w_{k}^{\prime}\right\}$ with permutation $\pi$. This gives $\mathcal{R}_{\text {w.e.c. }} \supseteq \mathcal{R}_{\mathrm{GNS}-\text { cut }}$ and completes the proof.

\section{V. $k$-GROUPCAST DIRECTED NETWORKS: SUM-RATE}

Definition. A $k$-groupcast directed network is a $k(k-1)$-unicast directed network $\mathcal{N}$ with $k$ distinct distinguished nodes (group-nodes) $v_{1}, v_{2}, \ldots, v_{k}$ with source-destination pairs $\left\{s_{(i, j)} ; d_{(i, j)}\right\}_{(i, j) \in \mathcal{I}}$ where $\mathcal{I}=\{(i, j): 1 \leq i, j \leq k, i \neq j\}$ and $s_{(i, j)}=v_{i}, d_{(i, j)}=v_{j}$.

Theorems 9 and 10 and Corollary 11 will refer to $k$-groupcast directed networks.

Theorem 9. (Naor-Zosin [18])

$$
\frac{1}{2} R_{\text {multicut }} \leq F_{\text {sum-rate }} \leq R_{\text {multicut }}
$$

Theorem 10.

$$
C_{\text {sum-rate }} \leq 2 R_{\text {multicut }}
$$

Theorems 9 and 10 together imply that routing flow is approximately capacity-achieving for sum-rate:

\section{Corollary 11.}

$$
\frac{1}{2} R_{\text {multicut }} \leq F_{\text {sum-rate }} \leq C_{\text {sum-rate }} \leq 2 R_{\text {multicut }}
$$

We give the proof of Theorem 10. We give the proof of Theorem 10 .

Proof:

Consider a $k$-groupcast directed network $\mathcal{N}$ with group-nodes $v_{1}, v_{2}, \ldots, v_{k}$. Let $E$ be a set of edges such that $\mathcal{G} \backslash E$ has no directed paths from $v_{i}$ to $v_{j}$ for each $(i, j) \in \mathcal{I}$. Let $\left(R_{(i, j)}:(i, j) \in \mathcal{I}\right) \in \mathcal{C}$. Observe that $E$ is a GNS-cut for source-destination pairs $\left\{s_{(i, j)} ; d_{(i, j)}\right\}_{(i, j) \in \mathcal{I}: i>j}$. Theorem 5 gives $\sum_{(i, j) \in \mathcal{I}: i>j} R_{(i, j)} \leq \sum_{e \in E} C_{e}$. Similarly, we can get $\sum_{(i, j) \in \mathcal{I}: i<j} R_{(i, j)} \leq \sum_{e \in E} C_{e}$. Adding, we obtain $\sum_{(i, j) \in \mathcal{I}} R_{(i, j)} \leq 2 \sum_{e \in E} C_{e}$, which completes the proof.

Remark 6. For the groupcast network in Fig. 3 with all edges having unit capacity, a simple XOR coding scheme achieves the rate tuple given by

$$
R_{(i, j)}= \begin{cases}1 & \text { if }(i, j)=(1,2) \text { or }(2,1) \\ 0 & \text { otherwise, }\end{cases}
$$

is achievable, while the multicut $R_{\text {multicut }}=1$. This shows that the factor 2 in the right most inequality of Theorem 10 cannot be improved upon. 


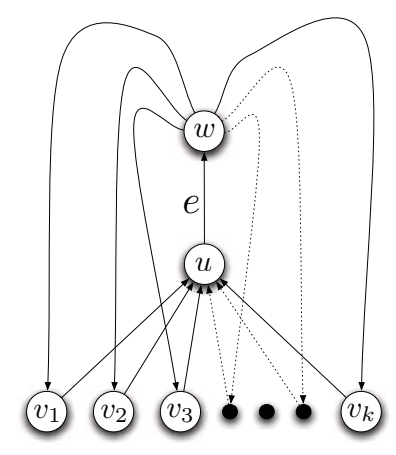

Fig. 3. Special groupcast network

\section{Gaussian Networks COMPOSEd OF BroAdCASt And Multiple ACCESs Channels}

A simplest model for a wireless network is a network that is comprised only of non-interacting multiple access channels and broadcast channels connected together through common nodes. This model is particularly interesting to study because the component channels are fully understood whereas a network composed of such channels is not well understood. We abbreviate networks composed only of multiple access channels and broadcast channels as MAC+BC networks. The communication network is represented by a directed graph $\mathcal{G}=(\mathcal{V}, \mathcal{E})$, and an edge coloring $\psi: \mathcal{E} \rightarrow C$, where $C$ is the set of colors. Each node $v$ has a set of colors $C(v) \subseteq C$ on which it operates. Each color can be thought of as an orthogonal resource, so that the broadcast and interference constraints for the wireless channel apply only within a given color. The set of edges $A_{c}$ corresponding to color $c$ interact with each other and can be said to constitute a channel.

The channel model can therefore be written as,

$$
y_{i}^{c}(t)=\sum_{j \in \ln _{c}(i)} h_{j i}^{c} x_{j}^{c}(t)+z_{i}^{c}(t) \quad \forall c \in C(i),
$$

where $x_{i}^{c}(t), y_{i}^{c}(t), z_{i}^{c}(t)$ are the transmitted vector, received vector, and noise vector on color $c$ at time instant $t, h_{j i}^{c}$ is the (fixed) channel coefficient between node $i$ and node $j$ on color $c$ and $\ln _{c}(i)$ represents the set of in-neighbors of node $i$ who are operating on color $c$. We denote by $y_{i}$ the vector comprised of $\left\{y_{i}^{c}\right\}$ for all $c \in C(i)$. We do not assume any symmetry in the channels, so that, in general $h_{i j}^{c}$ may be different from $h_{j i}^{c}$.

We say that a given $c \in C$ corresponds to a multiple access channel (MAC) of degree $d$, if the set of edges $A_{c}$ is of the form $A_{c}=\left\{i_{1} j, i_{2} j, \ldots, i_{d} j\right\}$, i.e., all edges are directed towards a particular node $j$. Similarly a channel $c$ is said to correspond to a broadcast channel (BC) of degree $d$ if $A_{c}=\left\{i j_{1}, i j_{2}, \ldots, i j_{d}\right\}$ for some node $i$. If $A_{c}$ is a singleton set, we say that the channel $c$ corresponds to an orthogonal link. A network is said to be a MAC+BC network if the set $C$ can be decomposed as $C=\mathcal{M} \cup \mathcal{B}$, where $\mathcal{M}$ is the set of MAC channels and $\mathcal{B}$ is the set of broadcast channels or orthogonal links. Stated alternately, a network is composed of broadcast and multiple access channels if and only if no edge is involved simultaneously in a broadcast and interference constraint inside the same color. We will call such a network a "Gaussian MAC+BC network". An example of such a network is shown in Fig. 4.

Each node $i$ has an average power constraint $P$ to transmit for each color that it transmits in. If there are distinct power constraints for different nodes, they can be absorbed into the channel co-efficient without loss of generality. We assume that the channel $h_{i j}^{c}$ is fixed (time-invariant) and is known at all the nodes.

\section{A. Multiple Unicast in Gaussian MAC+BC Networks}

A $k$-unicast Gaussian network has $k$ pairs of nodes $s_{i}, d_{i}, i=1,2, \ldots, k$, where node $s_{i}$ has a message to send to $d_{i}$ and $d_{i}$ has an independent message to send to $s_{i}$, both at rate $R_{i}$. We would like to characterize the closure of the set of all achievable rate tuples, called the capacity region $\mathcal{C}$. We will use $\mathcal{R}_{\text {ach }}$ to denote rates achievable by a simple scheme that we will propose. Also, for clarity, we will use $\mathcal{X}_{v}, \mathcal{Y}_{v}$ to denote the input and output alphabets at node $v$, implicitly understanding that in the Gaussian network, $\mathcal{X}_{v}=\mathbb{R}^{a}, \mathcal{Y}_{v}=\mathbb{R}^{b}$, for suitable integers $a$ and $b$. Formally, a $\left(\left\lceil 2^{T R_{1}}\right\rceil,\left\lceil 2^{T R_{2}}\right\rceil, \ldots,\left\lceil 2^{T R_{k}}\right\rceil, T\right)$ coding scheme for this network which communicates over $T$ time instants is comprised of the following.

1) Independent random variables $W_{i}$ which are distributed uniformly on $\mathcal{W}_{i}:=\left\{1, \ldots,\left\lceil 2^{T R_{i}}\right\rceil\right\}$ for $i=1, \ldots, k$ respectively. $W_{i}$ denotes the message intended from source $s_{i}$ to destination $d_{i}$.

2) The source mapping at source $s_{i}$ for time $t$,

$$
f_{s_{i}, t}:\left(\mathcal{W}_{i}, \mathcal{Y}_{s_{i}}^{t-1}\right) \rightarrow \mathcal{X}_{s_{i}}^{t} .
$$

3) The relay mappings for each $v \in \mathcal{V} \backslash\left\{s_{1}, d_{1}, s_{2}, d_{2}, \ldots, s_{k}, d_{k}\right\}$ and time $t$,

$$
f_{v, t}: \mathcal{Y}_{v}^{t-1} \rightarrow \mathcal{X}_{v}^{t}
$$




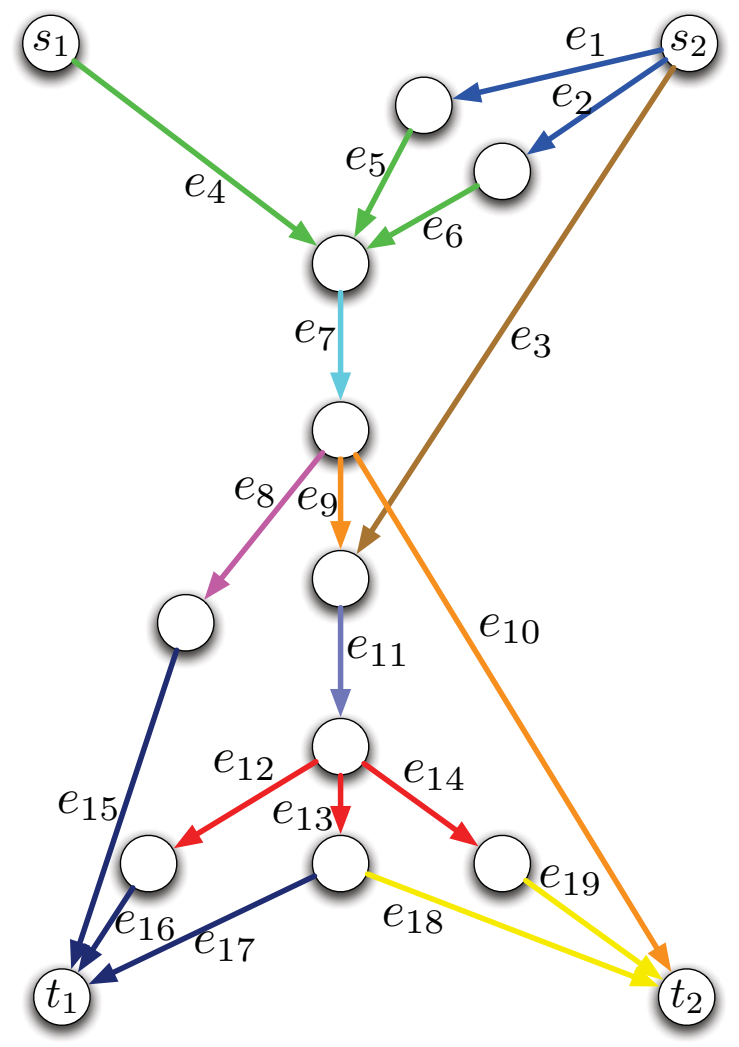

Fig. 4. Example of a MAC+BC Gaussian network

4) The decoding map at destination $d_{i}$,

$$
g_{d_{i}}: \mathcal{Y}_{d_{i}}^{T} \rightarrow \mathcal{W}_{i}
$$

If $\hat{W}_{i}$ is the decoded symbol at $d_{i}$, then the probability of error for destination $d_{i}$ under this coding scheme is given by

$$
P_{e}^{i}:=\operatorname{Pr}\left\{\hat{W}_{i} \neq W_{i}\right\} .
$$

A rate tuple $\left(R_{1}, R_{2}, \ldots, R_{k}\right)$, where $R_{i}$ is the rate of communication in bits per unit time from source $s_{i}$ to destination $d_{i}$, is said to be achievable if for any $\epsilon>0$, there exists a $\left(\left\lceil 2^{T R_{1}}\right\rceil,\left\lceil 2^{T R_{2}}\right\rceil, \ldots,\left\lceil 2^{T R_{k}}\right\rceil, T\right)$ scheme that achieves a probability of error lesser than $\epsilon$ for all nodes, i.e., $\max _{i} P_{e}^{i} \leq \epsilon$. The capacity region $\mathcal{C}$ is the closure of the set of all achievable rate tuples.

The capacity region can similarly be defined for the $k$-unicast problem with symmetric demands: For each $i=1,2, \ldots, k$, $s_{i}$ has a message to be communicated to $d_{i}$ at rate $R_{i}$ and $d_{i}$ has an independent message to be communicated to $s_{i}$ at the same rate $R_{i}$.

1) Weak edge-cut bound: Similar to the wireline network case, we define a weak edge-cut bound region for the wireless network with demand symmetry. The weak edge-cut bound region for the Gaussian MAC+BC network is defined by the following: consider any set of edges $F \subseteq \mathcal{E}$, and let $K(F)$ denote the set of $i \in\{1,2, \ldots, k\}$ such that either there is no path from $s_{i}$ to $d_{i}$ or there is no path from $d_{i}$ to $s_{i}$ in $\mathcal{G} \backslash F$. The value of the cut $F$ is defined by $\nu(F):=\sum_{c} \nu\left(F^{c}\right)$, where $F=\cup_{c} F^{c}$ with $F^{c}$ being the set of edges that participate in color $c$ and $\nu\left(F^{c}\right)$ is the capacity under complete coordination of source nodes in channel $c$. More formally, if $c$ is a broadcast channel, $\nu\left(F^{c}\right)$ is equal to the sum-capacity of the broadcast channel specified only by edges in $F^{c}$, under complete coordination of destination terminals in $F^{c}$. Similarly, if $c$ is a MAC channel, $\nu\left(F^{c}\right)$ is equal to the sum-capacity of the MAC channel specified by edges in $F^{c}$, under complete coordination of source terminals in $F^{c}$.

The weak edge-cut bound region is now given as

$$
\mathcal{R}_{\text {w.e.c. }}=\left\{\left(R_{1}, \ldots, R_{k}\right): \sum_{i \in K(F)} R_{i} \leq \nu(F) \forall F \subseteq \mathcal{E}\right\} .
$$

As in the wireline network case, it is not clear if $\mathcal{R}_{\text {w.e.c. }}$ is an outer bound to the capacity region $\mathcal{C}$.

Our main result is the following: 
Theorem 12. For the $k$-unicast problem with symmetric demands in a Gaussian MAC+BC network, the weak edge-cut bound is a fundamental outer bound on the capacity region and a simple separation strategy can achieve $\mathcal{R}_{\text {ach }}(P)$ which satisfies,

$$
\frac{\mathcal{R}_{\text {w.e.c. }}\left(\frac{P}{d_{\max }}\right)}{\kappa \log ^{3}(k+1)} \subseteq \mathcal{R}_{a c h}(P) \subseteq \mathcal{C}(P) \subseteq \mathcal{R}_{\text {w.e.c. }}(P),
$$

where $\kappa$ is a universal constant independent of problem parameters and $d_{\max }$ is the maximum degree of any broadcast or MAC component channel c.

Thus, the edge-cut bound is a fundamental upper bound on the capacity region and furthermore the edge-cut bound, scaled down in power by a factor $d_{\max }$ and scaled down in rate by a factor $\frac{1}{\kappa \log ^{3}(k+1)}$, can be achieved by the proposed scheme.

\section{B. Outer bound}

We first establish that the weak edge-cut bound is fundamental, i.e., every communication scheme must have rate pairs that lie inside this region: $\mathcal{C} \subseteq \mathcal{R}_{\text {w.e.c. }}$ We will prove this result using a GNS bound for Gaussian networks.

Given an $\ell$-unicast MAC+BC Gaussian network with source destination pairs $\left\{s_{i}, d_{i}\right\}_{i=1}^{\ell}$, we define a set of edges $F \subseteq \mathcal{E}$ to be a GNS-cut if there exists a permutation $\pi:\{1,2, \ldots, \ell\} \rightarrow\{1,2, \ldots, \ell\}$ such that there are no paths from $s_{i}$ to $d_{i}$ in $\mathcal{G} \backslash F$, whenever $\pi(i) \geq \pi(j)$.

Lemma 13. (GNS bound for MAC+BC Gaussian networks) For an l-unicast Gaussian MAC+BC network, every GNS cut F is fundamental, i.e., $\sum_{i \in K(F)} R_{i} \leq \nu(F)$ for any communication scheme achieving $\left(R_{1}, \ldots, R_{\ell}\right)$. Alternately $\mathcal{C} \subseteq \mathcal{R}_{\mathrm{GNS}-\mathrm{cut}}$, where

$$
\mathcal{R}_{\mathrm{GNS}-\mathrm{cut}}=\cap_{F \subseteq \mathcal{E}}\left\{\left(R_{1}, . ., R_{\ell}\right): \sum_{i \in K(F)} R_{i} \leq \nu(F)\right\} .
$$

An instance of Lemma 13 can be found in Fig. 5.

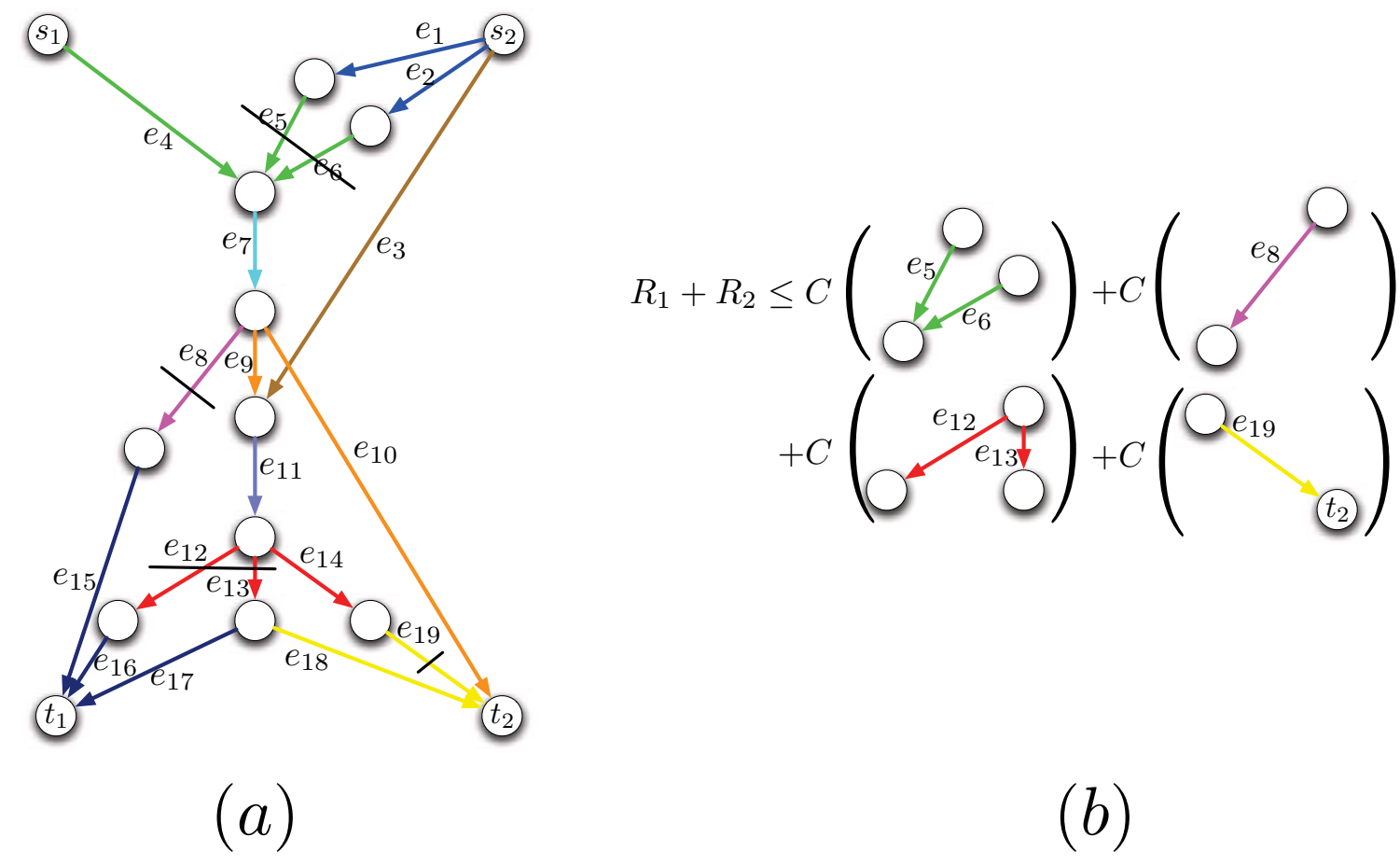

Fig. 5. GNS bound for MAC+BC Gaussian networks. The set of edges $\left\{e_{5}, e_{6}, e_{8}, e_{12}, e_{13}, e_{19}\right\}$ forms a GNS-cut in the two-unicast network of (a). The outer bound on the capacity region of the two-unicast network, that can be derived from the GNS-cut is shown in (b).

Proof: Let $F$ be a GNS-cut disconnecting $s_{i}$ from $d_{i}$ for $i=1,2, \ldots, \ell$ with say, the identity permutation $\pi_{\text {id }}$. Thus, $K(F)=\{1,2, \ldots, \ell\}$. A similar proof holds in the case when $K(F) \subset\{1,2, \ldots, \ell\}$. We first provide a proof of the GNS bound when the network has an acyclic underlying graph $\mathcal{G}$.

Recall that the set of colors $C=\mathcal{M} \cup \mathcal{B}$ where $\mathcal{M}$ consists of the colors of edges involved in MAC components and $\mathcal{B}$ consists of colors of edges involved in broadcast components or orthogonal links. For $\mu \in C$, let $A_{\mu}$ denote the set of edges involved in $\mu$. Now, construct a directed graph $\mathcal{G}^{\prime}$ as follows: for each $\mu \in C$, there is a node in $\mathcal{G}^{\prime}$ and add a directed edge 
from node $\mu$ to node $\nu$ in $\mathcal{G}^{\prime}$ if there exists an edge in $A_{\mu}$ that is upstream to some edge in $A_{\nu}$ in the original DAG $\mathcal{G}$. Since the set of all edges with a given color constitute either a MAC or a BC, we have that $\mathcal{G}^{\prime}$ is a directed acyclic graph. Thus, we can have a total order on the vertices of $\mathcal{G}^{\prime}$ consistent with the partial order of ancestry in $\mathcal{G}^{\prime}$. This gives a total order on $C$ and therefore also a total order on the subset $\mathcal{D}:=\left\{\mu \in C: F \cap A_{\mu} \neq \emptyset\right\}$, which we will denote by $\mu_{1}<\mu_{2}<\ldots<\mu_{r}$, where $\mu_{1}$ is the most "upstream".

- For $\mu \in \mathcal{M}$, we denote transmissions along edge $e$ in $A_{\mu}$ by $X_{e}$ and we denote the reception by $Y_{\mu}$ so that $Y_{\mu}=$ $\sum_{e \in A_{\mu}} X_{e}+Z_{\mu}$ where $Z_{\mu}$ is Gaussian noise. Further, define $U_{\mu}:=\left\{X_{e}: e \in F \cap A_{\mu}\right\}$, and $V_{\mu}:=\sum_{e \in F \cap A_{\mu}} X_{e}+Z_{\mu}$. Intuitively, $U_{\mu}\left(V_{\mu}\right)$ is the transmission (reception) on channel $\mu$ if only edges in $F$ were present in the channel.

- For $\mu \in \mathcal{B}$, we denote the transmission on the broadcast component or orthogonal link by $X_{\mu}$ and the receptions at heads of $e \in A_{\mu}$ by $Y_{e}$ so that $Y_{e}=X_{\mu}+Z_{e}$ where $\left\{Z_{e}, e \in A_{\mu}\right\}$ are independent Gaussian noise random variables. Further define $U_{\mu}:=X_{\mu}$, and $V_{\mu}:=\left\{Y_{e}: e \in F \cap A_{\mu}\right\}$.

Define $\tilde{Y}_{d_{i}}=\left\{Y_{\mu}^{n}: \operatorname{head}(e)=d_{i}, e \in A_{\mu}, \mu \in \mathcal{M}\right\} \cup\left\{Y_{e}^{n}: \operatorname{head}(e)=d_{i}, e \in A_{\mu}, \mu \in \mathcal{B}\right\}$.

$$
\begin{aligned}
& n\left[\sum_{i=1}^{\ell} R_{i}-\epsilon_{n}\right] \leq \sum_{i=1}^{\ell} I\left(W_{i} ; \tilde{Y}_{d_{i}}\right) \\
& \leq \sum_{i=1}^{\ell} I\left(W_{i} ;\left\{V_{\mu}^{n}: \mu \in \mathcal{D}\right\},\left\{W_{j}: j<i\right\}\right) \\
& \quad\left[\text { since } W_{i}-\left\{V_{\mu}^{n}: \mu \in \mathcal{D}\right\},\left\{W_{j}: j<i\right\}-\tilde{Y}_{d_{i}}\right. \\
& \quad \text { as } F \text { is a GNS cut with identity permutation] } \\
& =\sum_{i=1}^{\ell} I\left(W_{i} ;\left\{V_{\mu}^{n}: \mu \in \mathcal{D}\right\} \mid\left\{W_{j}: j<i\right\}\right) \\
& =I\left(\left\{W_{i}: 1 \leq i \leq \ell\right\} ;\left\{V_{\mu}^{n}: \mu \in \mathcal{D}\right\}\right) \\
& =h\left(\left\{V_{\mu}^{n}: \mu \in \mathcal{D}\right\}\right) \\
& \quad-h\left(\left\{V_{\mu}^{n}: \mu \in \mathcal{D}\right\} \mid\left\{W_{i}: 1 \leq i \leq \ell\right\}\right) \\
& \leq \sum_{\mu \in \mathcal{D}} h\left(V_{\mu}^{n}\right)-h\left(\left\{V_{\mu}^{n}: \mu \in \mathcal{D}\right\} \mid\left\{W_{i}: 1 \leq i \leq \ell\right\}\right) \\
& =: \sum_{\mu \in \mathcal{D}} h\left(V_{\mu}^{n}\right)-A .
\end{aligned}
$$$$
\text { as } F \text { is a GNS cut with identity permutation] }
$$

Now, we consider the negative term $A$ above.

$$
\begin{aligned}
& A=h\left(\left\{V_{\mu}^{n}: \mu \in \mathcal{D}\right\} \mid\left\{W_{i}: 1 \leq i \leq \ell\right\}\right) \\
& =h\left(V_{\mu_{1}}^{n}, V_{\mu_{2}}^{n}, \ldots, V_{\mu_{r}}^{n} \mid\left\{W_{i}: 1 \leq i \leq \ell\right\}\right) \\
& \geq h\left(V_{\mu_{1}}^{n}, V_{\mu_{2}}^{n}, \ldots, V_{\mu_{r}}^{n} \mid\left\{W_{i}: 1 \leq i \leq k\right\}, U_{\mu_{1}}^{n}\right) \\
& =h\left(V_{\mu_{1}}^{n} \mid\left\{W_{i}: 1 \leq i \leq \ell\right\}, U_{\mu_{1}}^{n}\right) \\
& \quad+h\left(V_{\mu_{2}}^{n}, \ldots, V_{\mu_{r}}^{n} \mid\left\{W_{i}: 1 \leq i \leq \ell\right\}, U_{\mu_{1}}^{n}, V_{\mu_{1}}^{n}\right) \\
& =h\left(V_{\mu_{1}}^{n} \mid U_{\mu_{1}}^{n}\right) \quad \\
& \quad+h\left(V_{\mu_{2}}^{n}, \ldots, V_{\mu_{r}}^{n} \mid\left\{W_{i}: 1 \leq i \leq \ell\right\}, U_{\mu_{1}}^{n}, V_{\mu_{1}}^{n}\right) \\
& \quad \quad \quad \text { since }\left\{W_{i}: 1 \leq i \leq \ell\right\}-U_{\mu_{1}}^{n}-V_{\mu_{1}}^{n} \\
& \left.\quad \text { as } \mu_{1} \text { is the most upstream channel }\right] \\
& \quad+h\left(V_{\mu_{1}}^{n} \mid U_{\mu_{1}}^{n}\right) \quad \\
& \quad+h\left(V_{\mu_{2}}^{n}, \ldots, V_{\mu_{r}}^{n} \mid\left\{W_{i}: 1 \leq i \leq \ell\right\}, U_{\mu_{1}}^{n}, V_{\mu_{1}}^{n}, U_{\mu_{2}}^{n}\right) \\
& =h\left(V_{\mu_{1}}^{n} \mid U_{\mu_{1}}^{n}\right)+h\left(V_{\mu_{2}}^{n} \mid\left\{W_{i}: 1 \leq i \leq \ell\right\}, U_{\mu_{1}}^{n}, V_{\mu_{1}}^{n}, U_{\mu_{2}}^{n}\right) \\
& \quad+h\left(V_{\mu_{3}}^{n}, \ldots, V_{\mu_{r}}^{n} \mid\left\{W_{i}: 1 \leq i \leq \ell\right\}, U_{\mu_{1}}^{n}, V_{\mu_{1}}^{n}, U_{\mu_{2}}^{n}, V_{\mu_{2}}^{n}\right) \\
& =h\left(V_{\mu_{1}}^{n} \mid U_{\mu_{1}}^{n}\right)+h\left(V_{\mu_{2}}^{n} \mid U_{\mu_{2}}^{n}\right) \\
& \quad+h\left(V_{\mu_{3}}^{n}, \ldots, V_{\mu_{r}}^{n} \mid\left\{W_{i}: 1 \leq i \leq \ell\right\}, U_{\mu_{1}}^{n}, V_{\mu_{1}}^{n}, U_{\mu_{2}}^{n}, V_{\mu_{2}}^{n}\right)
\end{aligned}
$$




$$
\begin{array}{r}
{\left[\begin{array}{l}
\text { since }\left\{W_{i}: 1 \leq i \leq \ell\right\}, U_{\mu_{1}}^{n}, V_{\mu_{1}}^{n}-U_{\mu_{2}}^{n}-V_{\mu_{2}}^{n} \\
\text { as only } \left.\mu_{1} \text { could be more upstream than } \mu_{2}\right]
\end{array}\right.} \\
\geq \sum_{\mu \in \mathcal{D}} h\left(V_{\mu}^{n} \mid U_{\mu}^{n}\right),
\end{array}
$$

from repeating these steps. Thus, we obtain

$$
\begin{aligned}
n\left[\sum_{i=1}^{\ell} R_{i}-\epsilon_{n}\right] & \leq \sum_{\mu \in \mathcal{D}} I\left(U_{\mu}^{n} ; V_{\mu}^{n}\right) \\
& \leq n \sum_{\mu \in \mathcal{D}} \nu\left(F_{\mu}\right) \\
& =n \nu(F),
\end{aligned}
$$

and therefore it follows that the GNS bound is a fundamental upper bound on the capacity region for acyclic networks.

For a general cyclic network, we can employ a standard time-layering argument in order to complete the proof. While the details of our method and the use of time-layering to deal with cyclic networks are fairly standard, see [2], [23], one key difference is that here we are using time-layering in order to prove an outer-bound, whereas the earlier works utilized time layering to show achievability. We will provide a brief sketch of the method here. Given a cyclic network $\mathcal{G}$ and a coding scheme over $n$ time instants, we construct a time-layered graph $\mathcal{G}^{n}$ as follows. The nodes in the graph $\mathcal{G}^{n}$ are arranged in $n+1$ layers $0,1, \ldots, n$. For each $i$, layer $i$ has a copy of all the nodes $V$ in the original graph, we call this $V[i]$ and the copy of node $v$ in layer i is called $v[i]$. Add directed edges in the graph in the following manner.

- For each $(u, v) \in \mathcal{E}$ in the original graph with channel coefficient $h_{v u}^{c}$ on color $c$, we add edges $(u[i], v[i+1])$ for $i=0,1, \ldots, n-1$ with channel coefficient $h_{v u}^{c_{i}}$ on color $c_{i}$.

- Create an edge from $v[i]$ to $v[i+1]$ for each $v$ of infinite capacity in an independent channel (in order to model memory of the link).

Thus the time-layered graph $\mathcal{G}^{n}$ is created. This graph defines an instance of a Gaussian MAC+BC network, which is acyclic. For this new graph, we define a communication problem by specifying that sources $s_{1}[0], \ldots, s_{\ell}[0]$ wish to communicate independent information to destinations $d_{1}[n], d_{2}[n], \ldots, d_{\ell}[n]$. Observe that any scheme on the original network utilizing $n$ time instants gives a valid scheme on this graph $\mathcal{G}^{n}$. Thus upper bounds on the communication rates in this graph serve as upper bounds to $n\left(R_{1}, R_{2}, \ldots, R_{\ell}\right)$ whenever $\left(R_{1}, R_{2}, \ldots, R_{\ell}\right)$ lies in the capacity region of the original network. Now given a GNS cut on the original graph with the identity permutation, defined by a set of edges $F$, we can define a cut on this graph $\mathcal{G}^{n}$ by $F^{n}:=\cup_{i \in[n]} F[i]$, where $F[i]=\left\{(u[i-1], v[i), \forall(u, v) \in F\}\right.$. If $F$ disconnected source $s_{a}$ from destination $d_{b}$ in the original graph, this cut $F^{n}$ disconnects $s_{a}[0]$ from $d_{b}[n]$ in the time-layered graph because any remaining path from $s_{a}[0]$ to $d_{b}[n]$ would imply a path in the original graph from $s_{a}$ to $d_{b}$. This implies that any GNS cut on the original graph can produce a GNS cut on $\mathcal{G}^{n}$ with $n$ times the value, as each edge occurs $n$ times in $F^{n}$. Since the rate is also scaled by $n$ times in this time layered graph, this proves that the GNS bound is a valid upper bound on the rate of an arbitrary (cyclic) graph.

Now that we know that GNS-cut is a valid upper bound on the Gaussian network for any $\ell$ unicast problem, we will define the GNS-cut for a symmetric demands problem (in exactly the same way as it was defined for wireline networks).

Definition. Given a $k$-pair unicast directed symmetric-demand MAC + BC Gaussian network with source-destination nodes $u_{1}, u_{2}, \ldots, u_{k}, v_{1}, v_{2}, \ldots, v_{k}$, we define the GNS-cut outer bound denoted by $\mathcal{R}_{\mathrm{GNS}-\mathrm{cut}}$, to be the set of all non-negative tuples $\left(R_{i}: 1 \leq i \leq k\right)$ that satisfy for every $F \subseteq \mathcal{E}(\mathcal{G})$, the inequality $\sum_{i \in J} R_{i} \leq \nu(F)$ whenever $F$ is a GNS-cut for $\left\{w_{1}, w_{2}, \ldots, w_{r} ; w_{1}^{\prime}, w_{2}^{\prime}, \ldots, w_{r}^{\prime}\right\}$ with some permutation $\pi$ where

- $J \subseteq\{1,2, \ldots, k\},|J|=r$

- $w_{1}, w_{2}, \ldots, w_{r}, w_{1}^{\prime}, w_{2}^{\prime}, \ldots, w_{r}^{\prime}$ are distinct,

- for $1 \leq j \leq r,\left(w_{j}, w_{j}^{\prime}\right)=\left(u_{i}, v_{i}\right)$ or $\left(v_{i}, u_{i}\right)$ for some $i \in J$.

By Theorem 6, we have that $\mathcal{R}_{\text {w.e.c. }}=\mathcal{R}_{\mathrm{GNS}-\text { cut }}$. Using this result in conjunction with Lemma 13, gives us the desired result,

$$
\mathcal{C} \subseteq \mathcal{R}_{\text {w.e.c. }}
$$

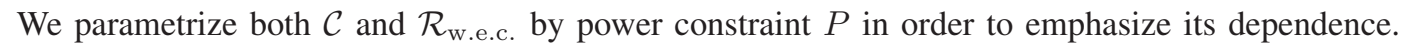

\section{Coding Scheme}

The coding scheme we propose is a separation-based strategy: each component broadcast or multiple access channel is coded for independently creating bit-pipes on which information is routed globally. In order to evaluate the rate region of this 
scheme, we use polymatroidal networks as an interface for which we can show that the flow region corresponding to routing and the bounding region defined by edge-cuts are close to each other. This coding scheme and the calculation of its achievable rate closely parallel the scheme proposed for networks composed of symmetric MAC and BC channels in [1].

For simplicity of notation, we will assume that all MAC and broadcast channels have degree $d=d_{\max }$. It will be clear from the details that this assumption is not necessary. For a finite set $V$, a set function $f: 2^{V} \mapsto \mathbb{R}$ is said to satisfy the polymatroidal axioms if

- $f(\emptyset)=0$

- $A \subseteq B \Longrightarrow f(A) \leq f(B)$,

- The function $f$ is submodular, i.e. for any two sets $A, B \subseteq V, f(A)+f(B) \geq f(A \cap B)+f(A \cup B)$.

A bounding region $\mathcal{B}$ defined over $\mathbb{R}^{|\mathbb{V}|}$ is said to be a polymatroidal region if it is of the form $\mathcal{B}=\left\{\left(R_{v}: v \in V\right): R_{v} \geq\right.$ 0 and for any $\left.A \subseteq V, \sum_{v \in A} R_{v} \leq f(A)\right\}$ for some functin $f$ that satisfies the polymatroidal axioms.

Let us first consider the coding for the multiple access channel with channel coefficients $h_{1}, \ldots, h_{d}$ and power constraint $P$ at each of the $d$ nodes. Let the rate region achievable on this multiple access channel be denoted by

$$
\mathcal{R}_{\text {ach }}^{\mathrm{MAC}}(P)=\left\{\bar{R}: \sum_{i \in A} R_{i} \leq \log \left(1+\sum_{i \in A}\left|h_{i}\right|^{2} P\right) \forall A\right\} .
$$

This region is known to be polymatroidal. The outer bound for the MAC under arbitrary source cooperation is given by

$$
\mathcal{R}_{\text {cut }}^{\mathrm{MAC}}(P)=\left\{\bar{R}: \sum_{i \in A} R_{i} \leq \log \left(1+\left(\sum_{i \in A}\left|h_{i}\right|\right)^{2} P\right) \forall A\right\} .
$$

The capacity region of a broadcast channel with channel coefficients $h_{1}, \ldots, h_{k}$ and power constraint $P$ is not a polymatroidal region. However, it can be approximated by a polymatroidal region [20]. In particular, the achievable region includes the following polymatroidal region, and we will restrict our broadcast channel scheme to operate inside the following polymatroidal region, as we will show that this is not too far from the cutset outer bound:

$$
\mathcal{R}_{\text {ach }}^{\mathrm{BC}}(P)=\left\{\bar{R}: \sum_{i \in A} R_{i} \leq \log \left(1+\sum_{i \in A}\left|h_{i}\right|^{2} \frac{P}{d}\right) \forall A\right\},
$$

The cutset bound on the broadcast channel under arbitrary destination cooperation is

$$
\mathcal{R}_{\text {cut }}^{\mathrm{BC}}(P)=\left\{\bar{R}: \sum_{i \in A} R_{i} \leq \log \left(1+\sum_{i \in A}\left|h_{i}\right|^{2} P\right) \forall A\right\} .
$$

It can be easily verified that $\mathcal{R}_{\text {cut }}^{\mathrm{MAC}}(P) \subseteq \mathcal{R}_{\text {ach }}^{\mathrm{MAC}}(d P)$ and $\mathcal{R}_{\text {cut }}^{\mathrm{BC}}(P) \subseteq \mathcal{R}_{\text {ach }}^{\mathrm{BC}}(d P)$. Now, each multiple access or broadcast channel can be replaced by a set of $d$ bit-pipes whose rates are jointly constrained by the corresponding capacity constraints. It turns out that this falls inside a class of networks called polymatroidal networks, that have been already studied [19]. We will now give a short description of polymatroidal networks and some results for these networks.

\section{Polymatroidal Networks}

Consider a directed graph $\mathcal{G}=(\mathcal{V}, \mathcal{E})$. We have considered networks in the previous chapter with capacity constraints on the edges. A polymatroidal network has more general capacity constraints coupling edges that meet at a node. In the polymatroidal network, for each node $v$ there are two associated submodular functions: $\rho_{v}^{\text {In }}$ and $\rho_{v}^{\text {Out }}$ which impose joint capacity constraints on the edges in $\ln (v)$ and $\operatorname{Out}(v)$ respectively. That is, for any set of edges $S \subseteq \ln (v)$, the total capacity available on the edges in $S$ is constrained to be at most $\rho_{v}^{\ln }(S)$. Similarly, $\rho_{v}^{\text {Out }}$ constrains edges in Out $(v)$.

For any subset of edges $F \subseteq \mathcal{E}$, we define the disconnection set $K(F)$ as the set of indices $i$ for which source $s_{i}$ has no paths to destination $d_{i}$ in $\mathcal{G} \backslash F$. In standard networks, the value of the cut $F$ is simply $\sum_{e \in F} c(e)$ where $c(e)$ is the capacity constraint on edge $e$. The value of a cut $F$ in polymatroidal networks is defined as follows: each edge $(u, v)$ in $F$ is first assigned to either $u$ or $v$; we say that an assignment of edges to nodes $g: F \rightarrow V$ is valid if it satisfies this restriction. Once this assignment is made, we can compute the value of the cut according to this assignment by evaluating the submodular functions corresponding to the set of edges grouped together. The value of the cut $\nu(F)$ is the minimum over all assignments, that is,

$$
\nu(F):=\min _{g: F \rightarrow V, g \text { valid }} \sum_{v}\left\{\rho_{v}^{\ln }\left(\ln (v) \cap g^{-1}(v)\right)+\rho_{v}^{\text {Out }}\left(\operatorname{Out}(v) \cap g^{-1}(v)\right)\right\} .
$$

A max-flow min-cut theorem for the single unicast problem in directed polymatroidal networks is known in the literature [24], [25]. For the $k$-unicast problem in a directed polymatroidal network with symmetric demands, the following theorem is 
proved in [19], which generalizes the result of [15] from edge capacity constraints to polymatroidal capacity constraints. The weak edge-cut bound for symmetric-demand polymatroidal networks is defined similarly as for standard networks.

Theorem 14. (from [19]) For a symmetric-demand directed polymatroidal network with $k$ source-destination pairs, any rate tuple in the weak edge-cut rate region divided by a factor $\kappa\left(\log ^{3}(k+1)\right)$ is achievable by routing, where $\kappa$ is a universal constant.

If $\mathcal{R}_{\text {w.e.c. }}^{\text {poly }}$ stands for the weak edge-cut region in the polymatroidal network and $\mathcal{R}_{\text {ach }}^{\text {poly }}$ stands for the region achievable by flow in the polymatroidal network, then Theorem 14 can be rewritten as:

$$
\frac{\mathcal{R}_{\text {w.e.c. }}^{\text {poly }}}{\kappa \log ^{3}(k+1)} \subseteq \mathcal{R}_{\text {ach }}^{\text {poly }} .
$$

\section{E. Analysis of Achievable Rates in Gaussian MAC+BC Network}

The proposed separation strategy converts the Gaussian MAC+BC network into a directed polymatroidal network with symmetric demands. Using the achievable rates for the corresponding MAC and BC channels from (34) and (36), we can see that this polymatroidal network has the following submodular functions at any given node $v$,

$$
\begin{aligned}
\rho_{v}^{\ln }(S) & =\sum_{c} \log \left(1+\sum_{(u v) \in S}\left|h_{v u}^{c}\right|^{2} P\right), \\
\rho_{v}^{\text {Out }}(S) & =\sum_{c} \log \left(1+\sum_{(v u) \in S}\left|h_{v u}^{c}\right|^{2} \frac{P}{d}\right) .
\end{aligned}
$$

This fully defines the polymatroidal network. Now any rate tuple achievable on this polymatroidal network is achievable in the Gaussian MAC+BC network using the proposed separation architecture.

Now we can use Theorem 14 to show that the achieved rate tuple in the Gaussian network is within a poly-logarithmic factor of the weak edge-cut bound in the polymatroidal network, i.e.

$$
\frac{\mathcal{R}_{\mathrm{w} . \text { e.c. }}^{\text {poly }}(P)}{\kappa \log ^{3}(k+1)} \subseteq \mathcal{R}_{\text {flow }}^{\text {poly }}(P)=\mathcal{R}_{\text {ach }}^{\mathrm{g}}(P) .
$$

Here we have parametrized all the rate regions by the power constraint in order to make this dependence explicit. In order to prove our main result, we still need to connect the weak edge-cut bound in the polymatroidal network to the weak edge-cut bound in the Gaussian network.

We will connect the value of the cut in the polymatroidal network to the value of the cut in the Gaussian network. Let us take a cut derived from a set of edges $F$ in the polymatroidal network and a valid assignment $g$ of $F$, which yields the minimum among all possible valid assignments. In this assignment, each edge $(u, v)$ of $F$ is assigned either to $u$ or to $v$. Thus for any node, some incoming edges are assigned together and some outgoing edges are assigned together and the value of the cut is given by

$$
\nu(F)=\sum_{v}\left\{\rho_{v}^{\ln }\left(\ln (v) \cap g^{-1}(v)\right)+\rho_{v}^{\text {Out }}\left(\operatorname{Out}(v) \cap g^{-1}(v)\right)\right\} .
$$

Note that each of these functions $\rho_{v}^{\ln }$ and $\rho_{v}^{\text {Out }}$ corresponds to the constraints in the achievable region of the original MAC and broadcast channels. If we take the cut corresponding to $F$ in the original network, then these functions will be replaced by the functions corresponding to the cut in the MAC and broadcast channels, whose equations are given in (35) and (37). It has been observed earlier that,

$$
\begin{aligned}
\mathcal{R}_{\text {cut }}^{\mathrm{MAC}}(P) & \subseteq \mathcal{R}_{\mathrm{ach}}^{\mathrm{MAC}}(d P) \\
\mathcal{R}_{\text {cut }}^{\mathrm{BC}}(P) & \subseteq \mathcal{R}_{\text {ach }}^{\mathrm{BC}}(d P) .
\end{aligned}
$$

Note that the value of any cut in the polymatroidal network is a function of the power constraint implicitly, since $\rho^{\ln }$ and $\rho^{\text {Out }}$ are functions of the power constraint. Let us call this function $v(P)$. Now if we look at the same cut in the Gaussian network then the value of this cut here is at most $v(d P)$ because of (43) and (44). Thus the weak edge-cut region in the polymatroidal network and the weak edge-cut region in the Gaussian network can be related to each other as follows,

$$
\mathcal{R}_{\text {w.e.c. }}^{\mathrm{g}}(P) \subseteq \mathcal{R}_{\text {w.e.c. }}^{\text {poly }}(d P),
$$

or alternately $\mathcal{R}_{\mathrm{w} . e . c .}^{\mathrm{g}}\left(\frac{P}{d}\right) \subseteq \mathcal{R}_{\text {w.e.c. }}^{\text {poly }}(P)$. This result, when combined with (42) and (33), yields the following relationship,

$$
\frac{\mathcal{R}_{\mathrm{w} . \text {.e.c. }}^{\mathrm{g}}\left(\frac{P}{d}\right)}{\kappa \log ^{3}(k+1)} \subseteq \mathcal{R}_{\mathrm{ach}}^{\mathrm{g}}(P) \subseteq \mathcal{C} \subseteq \mathcal{R}_{\text {w.e.c. }}^{\mathrm{g}}(P),
$$

which completes the proof of Theorem 12 . 


\section{General Gaussian Networks}

In this section, we consider general Gaussian networks, i.e., networks where broadcast and MAC can occur simultaneously. Our basic idea will remain similar to what we did in the case of the Gaussian MAC+BC network, where we employed a separation strategy. A good physical layer scheme that approximately achieved the cut-set bound converted the MAC $+\mathrm{BC}$ Gaussian network into a polymatroidal network on which routing was shown to be approximately optimal.

In order to provide guarantees on the performance of our layered architecture in general Gaussian networks, we need physical layer schemes that achieve close to the cut-set bound on the component channels. This class of networks includes as a special case the interference channel and the $X$-channel (where every transmitter has a message to transmit to every receiver). In a general Gaussian network, it is not clear what a component channel is. In [1], it was identified that the $X$-channel can be viewed as a basic component channel of a general Gaussian network. Good communication schemes are known for the $X$ channel under the following scenarios:

1) Degrees-of-freedom in fixed Gaussian channels

2) Capacity approximation in ergodic Gaussian channels.

As such, our network-level results also apply under these two settings.

\section{A. Fixed Gaussian channels}

The communication network is represented by a directed graph $\mathcal{G}=(\mathcal{V}, \mathcal{E})$. The edges $(j, i)$ that are present have fading coefficients $h_{i j}$ on them, each of which are chosen independently from a continuous fading distribution. Note that in a general Gaussian network, the presence of edge $(j, i)$ does not necessarily imply the presence of edge $(i, j)$. There are $k$ pairs of specially designated nodes $s_{i}, d_{i}$ such that $s_{i}$ has a message to send to $d_{i}$ at rate $R_{i}$ and $d_{i}$ has an independent message to send to $s_{i}$ at the same rate $R_{i}$. The channel model can be written as

$$
y_{i}(t)=\sum_{j \in \ln (i)} h_{i j} x_{j}(t)+z_{i}(t) \forall t=1,2, \ldots, T,
$$

where $x_{i}(t), y_{i}(t), z_{i}(t)$ are the transmitted vector, received vector, and noise vector at time $t$, and $\ln (i)$ represents the set of neighbors of node $i$ who have an incoming edge to $i$. The noise vector is assumed to have unit variance and is independent at each node. There is an average power constraint of $P$ per node. The degrees-of-freedom (DOF) tuple $\left(d_{1}, \ldots, d_{k}\right)$ is said to be achievable if, for each $P$, there is a scheme achieving rate tuple $\left(R_{1}(P), \ldots, R_{k}(P)\right)$ such that $d_{i}=\lim _{P \rightarrow \infty} \frac{R_{i}}{\log P}$. The closure of the set of all achievable DOF tuples is called the DOF region $\mathcal{D}$. Let $\mathcal{D}_{\text {ach }}$ denote the DOF tuples achievable by our specific strategy and let $\mathcal{D}_{\text {w.e.c. }}$ correspond to the weak edge-cut bound (which is defined formally below).

1) Weak Edge-Cut Bound:: In order to define the weak edge-cut bound in the general Gaussian network case, we will utilize the weak edge-cut bound region in the Gaussian MAC+BC network case. We will observe that there are several ways to upper bound the capacity of a given general Gaussian network by a related network of Gaussian MAC+BC channels. This can be done by "deactivation" of some of the MAC and BC constraints. Formally, consider a coloring on the set of edges such that the channel corresponding to each color is a MAC or broadcast channel. This coloring produces a Gaussian MAC+BC network whose DOF region contains the DOF region of the original network, as we shall show. For a MAC+BC network, each orthogonal MAC or $\mathrm{BC}$ component contributes a DOF of 1 . Thus, the DOF value of the cut in the colored network is the total number of MAC or BC channels involved in the cut. Now, we define $d(F)$ as the tightest bound obtainable on the DOF by bounding it based on any suitable coloring giving a MAC+BC network. Finally, define $\mathcal{D}_{\text {w.e.c. }}=\left\{\left(d_{1}, \ldots, d_{k}\right): \sum_{i: i \in K(F)} d_{i} \leq d(F) \forall F \subseteq \mathcal{E}\right\}$. It is not clear, as earlier, that $\mathcal{D}_{\text {w.e.c. }}$ is a fundamental upper bound on any achievable DOF tuple under symmetric demands.

Our main result is the following.

Theorem 15. For a directed wireless network with symmetric demands, if the fixed channel coefficients are drawn from a continuous distribution, the DOF region given by $\mathcal{D}_{\text {ach }}$ satisfying

$$
\frac{\mathcal{D}_{\text {w.e.c. }}}{\kappa \log ^{3}(k+1)} \subseteq \mathcal{D}_{\text {ach }} \subseteq \mathcal{D} \subseteq \mathcal{D}_{\text {w.e.c. }},
$$

is achievable, with probability 1.

2) Coding Scheme: Our coding scheme involves a conversion of the Gaussian network into a bit-pipe network (more specifically, a polymatroidal network) and then routing on this induced polymatroidal network. This coding scheme and the analysis of the achievable rate closely parallel the scheme proposed for general wireless networks in [1]. However, the outer bound is a little more interesting because we do not currently have a GNS bound for general wireless networks, and have written it only for $\mathrm{MAC}+\mathrm{BC}$ networks. We use the bound for $\mathrm{MAC}+\mathrm{BC}$ networks to obtain a bound for general wireless networks.

First, consider an interference channel with $l$ sources $s_{1}, s_{2}, \ldots, s_{k}$, wishing to communicate to their respective destinations $d_{1}, d_{2}, \ldots, d_{k}$. Suppose that the connectivity between sources and destinations is described by a suitable bipartite graph and 
that for each $i$, there is an edge from $s_{i}$ to $d_{i}$. Then, it is well-known that the real interference alignment scheme [26] achieves half DOF per transmitter-receiver pair simultaneously, almost surely over the randomness in the channel co-efficients.

In our Gaussian network, we choose two subsets of nodes of equal size. We choose one set to be a set of "transmitter" nodes and the other set to be a set of "receiver" nodes. This creates for us an interference channel. We choose a suitable matching in the connectivity from the transmitters to the receivers to give us the transmitter-receiver pairs. This creates bit pipes that offer half DOF for each transmitter-receiver pair. We then time-share between all possible choices of subset pairs and over all possible matchings.

It has been shown in [1] that such a choice in fact leads to a simple polymatroidal network with the same set of of edges as the original Gaussian network, and with the following sub modular functions at each node, almost surely

$$
\begin{aligned}
\rho_{v}^{\ln }(S) & =\frac{1}{2} \quad \forall S \neq \emptyset \subseteq \ln (v), \forall v \in V, \\
\rho_{v}^{\text {Out }}(T) & =\frac{1}{2} \quad \forall T \neq \emptyset \subseteq \operatorname{Out}(v), \forall v \in V .
\end{aligned}
$$

Now, on this polymatroidal network, information is routed from the source nodes to the destination nodes. Since the traffic demand is symmetric between $s_{i}$ and $d_{i}$, the result of Theorem 14 shows that the rate achieved on the polymatroidal network is within a poly-logarithmic factor of the edge-cut bound in the polymatroidal network, i.e.,

$$
\frac{\mathcal{R}_{\mathrm{w} . \text { e.c. }}^{\text {poly }}}{\kappa \log ^{3}(k+1)} \subseteq \mathcal{R}_{\text {ach }}^{\text {poly }} .
$$

Observe that if $\left(R_{1}, \ldots, R_{k}\right) \in \mathcal{R}_{\text {ach }}^{\text {poly }}$ is achievable in the polymatroidal network, a degrees of freedom tuple $\left(D_{1}, \ldots, D_{k}\right)=$ $\left(R_{1}, \ldots, R_{k}\right)$ is achievable almost surely in the Gaussian network using the strategy described above. Thus $\mathcal{D}_{\text {ach }}^{\mathrm{g}}=\mathcal{R}_{\text {ach }}^{\text {poly }}$. So, (51) gives

$$
\frac{\mathcal{R}_{\text {w.e.c. }}^{\text {poly }}}{\kappa \log ^{3}(k+1)} \subseteq \mathcal{R}_{\text {ach }}^{\text {poly }}=\mathcal{D}_{\text {ach }}^{\mathrm{g}} .
$$

Now, we need to connect $\mathcal{R}_{\mathrm{w} . e . c .}^{\text {poly }}$ to $\mathcal{D}_{\mathrm{w} \text {.e.c. }}^{\mathrm{g}}$ in order to obtain the desired result. For this, we want to find the DOF value of a cut in the Gaussian network. We upper bound the DOF in the original Gaussian network by creating a modified network. We will color the edges in a certain way such that broadcast and superposition constraints are active only inside a color (similar to the coloring defined for MAC+BC networks (see Sec. VI).

We need the following lemma, which relates the rates achieved in the two networks.

Lemma 16. (from [1]) If we color the edges in the Gaussian network to obtain a "colored network", then the capacity regions of the two networks are related by the following:

$$
\mathcal{R}_{a c h}^{g}(P) \subseteq \mathcal{R}^{g}(P) \subseteq \mathcal{R}^{c o l}(d P),
$$

where the superscript ${ }^{g}$ is used for the original Gaussian network and the superscript ${ }^{c o l}$ is used to denote the colored Gaussian network and d is the maximum degree of any node in the network, that is, $\mathcal{R}_{\text {ach }}^{g}(P)$ is the set of rate tuples achieved by our scheme, $\mathcal{R}^{g}(P)$ is the capacity region and $\mathcal{R}^{\text {col }}(d P)$ is the capacity region of the colored Gaussian network at power constraint $d P$.

Now, if the coloring yields a MAC+BC network, Lemma 13 informs us that the GNS bound is a fundamental outer bound on the capacity region. Also, from Theorem 6 for the symmetric demands problem, the weak edge cut bound is identical to the GNS bound, that is,

$$
\mathcal{R}^{\mathrm{col}}(P) \subseteq \mathcal{R}_{\text {w.e.c. }}^{\mathrm{col}}(P)
$$

(53) and (54) imply

$$
\mathcal{R}_{\text {ach }}^{\mathrm{g}}(P) \subseteq \mathcal{R}^{\mathrm{g}}(P) \subseteq \mathcal{R}^{\mathrm{col}}(d P) \subseteq \mathcal{R}_{\text {w.e.c. }}^{\text {col }}(d P)
$$

Since degrees of freedom do not change by constant factor power scaling, we get

$$
\mathcal{D}_{\text {ach }}^{\mathrm{g}} \subseteq \mathcal{D}^{\mathrm{g}} \subseteq \mathcal{D}^{\text {col }} \subseteq \mathcal{D}_{\text {w.e.c. }}^{\text {col }}
$$

This justifies that $\mathcal{D}_{\text {w.e.c. }}^{\text {col }}$ is indeed an upper bound on the DOF of the original network. From the definition of $\mathcal{D}_{\text {w.e.c. }}^{g}$, we have $\mathcal{D}_{\text {w.e.c. }}^{\mathrm{g}}=\cap_{\text {col } \in \mathcal{Z}} \mathcal{D}_{\text {w.e.c. }}^{\text {col }}$, where $\mathcal{Z}$ denotes the set of all possible colorings that yield MAC+BC Gaussian networks. Using this and (56) we have

$$
\mathcal{D}_{\text {ach }}^{\mathrm{g}} \subseteq \mathcal{D}^{\mathrm{g}} \subseteq \mathcal{D}_{\text {w.e.c. }}^{\mathrm{g}} \text {. }
$$

Now, consider a subset of edges $F \subseteq \mathcal{E}$ in the polymatroidal network. Let the value of this cut in the polymatroidal network be $\nu(F)$. By the definition of cuts in polymatroidal network, $\nu(F)$ is achieved by an optimal assignment $g$ of each edge 
$(u, v) \in F$ to either node $u$ or $v$ and the summing up the sub-modular functions in the polymatroidal network. Recall that the value of the cut $\nu(F)$ is given by,

$$
\nu(F):=\sum_{v}\left\{\rho_{v}^{\ln }\left(\ln (v) \cap g^{-1}(v)\right)+\rho_{v}^{\text {Out }}\left(\operatorname{Out}(v) \cap g^{-1}(v)\right)\right\} .
$$

Each sub-modular function has the same value $\left(\frac{1}{2}\right)$ for a non-empty subset. Hence the total value of the edge-cut in the polymatroidal network is equal to one half of the number of nodes that are assigned at least one incoming edge by the assignment plus one half of the number of nodes that are assigned at least one outgoing edges. From this optimal assignment $g$, we describe a specific coloring from a partition of $F$ into equivalence classes using the following equivalence relation $\sim$, where

$$
\begin{aligned}
e_{1} \sim e_{2} \Longleftrightarrow \quad g\left(e_{1}\right)=g\left(e_{2}\right)=\operatorname{head}\left(e_{1}\right)=\operatorname{head}\left(e_{2}\right) \text { or } \\
g\left(e_{1}\right)=g\left(e_{2}\right)=\operatorname{tail}\left(e_{1}\right)=\operatorname{tail}\left(e_{2}\right),
\end{aligned}
$$

i.e., $e_{1} \sim e_{2}$ if they are assigned by $g$ to the same node and share the head or tail. We assign distinct color to equivalence classes and each edge in the equivalence class is assigned the same color. The edges not in $F$ are all assigned distinct colors, which are disjoint from the colors already assigned. This defines a colored network, whose DOF upper bounds the DOF of the original network.

The DOF value of this GNS cut is simply the sum of the number of broadcast or MAC channels involved in the cut. This is exactly equal to the number of colors in the cut, which is the same as the number of distinct equivalence classes, which is equal to $2 \nu(F)$. Thus the degrees of freedom of the cut $F, d(F)$ is upper bounded by $2 \nu(F)$, and therefore,

$$
\mathcal{D}_{\text {w.e.c. }}^{\mathrm{g}} \subseteq \mathcal{D}_{\text {w.e.c. }}^{\text {col }} \subseteq 2 \mathcal{R}_{\text {w.e.c. }}^{\text {poly }} \text {. }
$$

From (52), (57) and (58), we get

$$
\frac{\mathcal{D}_{\mathrm{w} . e . c .}^{\mathrm{g}}}{\kappa \log ^{3}(k+1)} \subseteq \mathcal{D}_{\text {ach }}^{\mathrm{g}} \subseteq \mathcal{D}^{\mathrm{g}} \subseteq D_{\text {w.e.c. }}^{\mathrm{g}}
$$

which proves our claim.

\section{B. Ergodic Gaussian Channels}

In an ergodic wireless network, the channel model is similar to the fixed Gaussian network, except that instead of assuming constant channel coefficients $h_{i j}(t)=h_{i j}$, we assume that for each $(j, i) \in \mathcal{E}$, the channel coefficient $h_{i j}(t)$ is varying as a function of time. In particular, we assume that $h_{i j}(t)$ is i.i.d. according to a fading distribution $p(h)$ across $i, j$, $t$, i.e., identical and independent across all the edges and time. We make another assumption, that the fading distribution $p(h)$ is symmetric, i.e., $p(h)=p(-h)$ with $\mathbb{E}_{h}|h|^{2}=1$ and also that it satisfies the following weak tail assumption,

$$
a:=e^{-\mathbb{E}\left(\log |h|^{2}\right)}<\infty .
$$

Many common fading distributions satisfy this assumption. One particularly useful example of such a fading distribution is the i.i.d. complex Gaussian distribution, for which $a \approx 1.78$ [27].

Our main result in this setting is the following.

Theorem 17. For directed Gaussian network with symmetric demands, with a symmetric weak-tailed ergodic fading distribution, the rate region given by $\mathcal{R}_{\text {ach }}(P)$ satisfying

$$
\frac{\mathcal{R}_{\text {w.e.c. }}\left(\frac{P}{a d^{3}}\right)}{\kappa \log ^{3}(k+1)} \subseteq \mathcal{R}_{a c h}(P) \subseteq \mathcal{C}(P) \subseteq \mathcal{R}_{\text {w.e.c. }}(P),
$$

is achievable, where $\kappa$ is a universal constant, $d$ is the maximum degree of any node and $a=e^{-\mathbb{E}\left(\log |h|^{2}\right)}$.

The proof of this result is similar to the case of general static networks in Subsection VII-A and has been carried out for bidirected wireless networks in [1]. We only sketch the basic differences in the proof here.

The wireless network is converted into a polymatroidal network using the following physical layer scheme: At any given instant, we choose a subset of nodes as transmitter and another subset of nodes of the same size as receiver, and pair them up as into transmitter-receiver pairs. This creates an interference channel between the set of transmitting nodes and receiving nodes, in this interference channel, ergodic interference alignment [28] can achieve the following rate,

$$
r:=\frac{1}{2} \mathbb{E}_{h} \log \left(1+|h|^{2} P\right),
$$


for every transmitter receiver pair which is connected. When we time-share between all such possible transmitter subset, receiver subset and matching choices, we get a polymatroidal network specified by the same set of edges as the original wireless network with submodular functions on the nodes given by,

$$
\begin{aligned}
\rho_{v}^{\ln }(S) & =r \quad \forall S \neq \emptyset \subseteq \ln (v) \forall v \in V, \\
\rho_{v}^{\text {Out }}(T) & =r \quad \forall T \neq \emptyset \subseteq \operatorname{Out}(v) \forall v \in V .
\end{aligned}
$$

We define the weak edge-cut for the ergodic wireless network similar to the definition of the weak edge-cut for fixed wireless network, using the sharpest upper bound given by the coloring of the edges that induces a MAC+BC network, i.e.,

$$
\mathcal{R}_{\mathrm{w} . e . c .}^{\mathrm{g}}(P)=\cap_{\text {col } \in \mathcal{Z}} \mathcal{R}_{\mathrm{w} . \text { e.c. }}^{\text {col }}(d P),
$$

where the superscript 'col' here denotes a particular instance of a colored network and $\mathcal{Z}$ is the set of all possible colorings of the network that lead to a MAC+BC network. By Lemma 16 and Lemma 13, we know that $\mathcal{C}(P) \subseteq \mathcal{R}_{\mathrm{w} . e . c .}^{\mathrm{g}}(P)$, i.e., the region $\mathcal{R}_{\mathrm{w} . e . c .}^{\mathrm{g}}(P)$ is a fundamental upper bound for the capacity region.

We then perform routing on this polymatroidal network from the sources to the sinks in the given traffic model. By Theorem 14, routing can achieve rate tuples close to the cut-set bound in the polymatroidal network to within a poly-logarithmic factor,

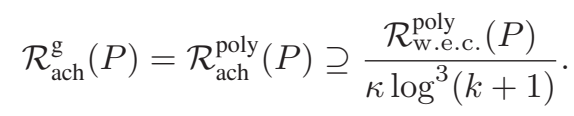

In order to connect the cuts of the Gaussian network to the polymatroidal network, we take any edge-cut $F$ and the optimizing assignment $g$ of the cut, and color the edges of the Gaussian network using equivalence classes of $F$, in exactly the same way as in the fixed Gaussian network case. The cuts in the colored network can be related to the cuts in the polymatroidal network, along the lines of [1] as follows:

$$
\mathcal{R}_{\text {w.e.c. }}^{\text {col }}(P) \subseteq \mathcal{R}_{\text {w.e.c. }}^{\text {poly }}\left(a d^{2} P\right) .
$$

Therefore, we get,

$$
\mathcal{R}_{\mathrm{w} . \text { e.c. }}^{\mathrm{g}}(P) \subseteq \mathcal{R}_{\mathrm{w} . \text { e.c. }}^{\text {poly }}\left(a d^{3} P\right),
$$

which completes the sketch of proof of Theorem 17.

\section{BRoAdCAST ERASURE NETWORKS With FEEDBACK}

In this section, we consider broadcast erasure networks in the presence of local one-bit-per-packet feedback. Unicast in broadcast erasure networks is well understood, for which it has been shown [29] that min-cut is achievable using a global linear network coding scheme (in [30], it is shown that knowledge of erasure locations is not necessary at the destination).

First we define a broadcast erasure channel as a packet transmission channel where a transmitter can transmit packets and there are $d$ receivers connected to the transmitter, each of which either receives the packet or the packet gets erased. In this channel, the source node has independent messages $W_{1}, \ldots, W_{d}$ to communicate to each of the destinations $T_{1}, . ., T_{d}$ at rate $R_{1}, . ., R_{d}$.

Formally, for a broadcast erasure channel with $d$ receivers, the channel model can be written as,

$$
y_{i}(t)=e_{i}(t) x(t), \quad \forall i=1,2, \ldots, d, \forall t,
$$

where $e_{i}$ is a binary random variable which when 0 represents that at receiver $i$, the packet got erased (we can think of $x \in \mathcal{X}$, where $\mathcal{X}=\{1,2, \ldots, M\}$. If $e_{i}$ are all independent, then the broadcast erasure channel is said to be an independent erasure broadcast channel. Such a channel is specified by erasure probabilities $\epsilon_{i}, i=1,2, \ldots, d$, where

$$
\epsilon_{i}=\operatorname{Pr}\left\{e_{i}=0\right\} .
$$

For the purpose of simplicity in this paper we will consider only broadcast erasure channels that are independent and symmetric, which implies that there is only one parameter $\epsilon$ and $\epsilon_{i}=\epsilon \quad \forall i$. For this channel, the cut region is specified as follows,

$$
\mathcal{R}_{\text {cut }}:=\left\{\left(R_{1}, . ., R_{d}\right): \sum_{i \in S} R_{i} \leq 1-\epsilon^{|S|} \quad \forall S \subseteq[d]\right\} .
$$

The capacity region is given by

$$
\mathcal{R}_{\text {ach }}:=\left\{\left(R_{1}, . ., R_{d}\right): \sum_{i \in[d]} R_{i} \leq 1-\epsilon\right\} .
$$

The sum capacity is given by $1-\epsilon$, whereas the sharpest sum-rate bound implied by the cut is $1-\epsilon^{d}$. Thus the sum capacity is off by a factor $\frac{1-\epsilon^{d}}{1-\epsilon} \rightarrow d$ as $\epsilon \rightarrow 1$ and the cut is a very bad approximation of the capacity region.

http://mc.manuscriptcentral.com/t-it 
A broadcast erasure channel is said to have feedback if the transmitter gets to know the realization of $e_{i}(t-1) \forall i=1,2, \ldots, d$ at time $t$. For a broadcast erasure channel with feedback, the following result connects $\mathcal{R}_{\text {ach,fb }}$, the achievable rate in the presence of feedback to the value of the cut (which continues to bound the information rate even in the presence of feedback).

Lemma 18. [31]-[33]

$$
\begin{gathered}
R_{a c h, f b}=\left\{\left(R_{1}, \ldots, R_{D}\right) \mid \sum_{i=1,2, . ., d} \frac{R_{\pi(i)}}{1-\epsilon^{i}} \leq 1 \quad \forall \pi\right\} . \\
\mathcal{R}_{a c h, f b} \supseteq \frac{\mathcal{R}_{c u t}}{\psi(\epsilon, d)},
\end{gathered}
$$

where

$$
\frac{1}{\psi(\epsilon, d)}:=\sum_{i=1}^{d} \frac{\epsilon^{i-1}-\epsilon^{i}}{1-\epsilon^{i}} \geq \frac{1}{\log _{2}(d+1)} .
$$

\section{A. Broadcast erasure network with feedback}

Consider a network comprised of broadcast erasure channels with feedback. The network is described by a directed graph $\mathcal{G}=(V, E)$. Let there be $C$ such channels in the network. Let $x_{v}^{c}$ denote the input to the broadcast erasure channel $c$ at node $v$ (if node $v$ does not possess that channel $c$, then $x_{v}^{c}=\emptyset$ ) and similarly let $y_{v}^{c}$ denote the output from broadcast channel $c$ at node $v$. There is a symmetric demands model: there are $k$ pairs of nodes $s_{i}, t_{i}, i=1,2, \ldots, k$, where $s_{i}$ wants to communicate with $t_{i}$ at rate $R_{i}$ and similarly, $t_{i}$ wants to communicate with $t_{i}$ at the same rate $R_{i}$.

We use $\mathcal{R}_{\text {ach }}^{\text {er }}$ to denote the achievable rate region, where er is the superscript used to denote 'erasure'. For this network, the main result we can show is the following,

Theorem 19. For a broadcast erasure network with feedback and symmetric demands, a separation strategy can achieve a rate given by $\mathcal{R}_{\text {ach }}$, where,

$$
\frac{\mathcal{R}_{\text {w.e.c. }}^{e r}}{\kappa \psi(\epsilon, d) \log ^{3}(k+1)} \subseteq \mathcal{R}_{a c h}^{e r} \subseteq \mathcal{R}_{\text {w.e.c. }}
$$

with $\kappa$ a universal constant and $d$ the maximum degree of any broadcast channel in the network and $\psi(\epsilon, d) \leq \log _{2}(d+1)$.

The proof of this result is simiar to that of the MAC+BC networks, and therefore the proof is only briefly sketched here.

- On each broadcast erasure channel, use the scheme of Lemma 18 to achieve a rate region close to the cut. We use the reduced region for the achievable scheme given by $\frac{\mathcal{R}_{\text {ch }}^{\text {ch }}}{\log _{2}(d+1)}$, where $\mathcal{R}_{\text {cut }}^{\text {ch }}$ is the cut bound of the particular channel ch. The reason for doing this is that this region is a polymatroidal region, whereas the original achievable region in equation (73) is not.

- This implies that the broadcast erasure network can be replaced by a bit-pipe network, where the capacity of the bit-pipes are jointly constrained.

- This network is not directly a polymatroidal network, on which routing achieves within a poly-logarithmic factor of the cut-set bound in the polymatroidal network.

- Now, any cut in the polymatroidal network has a corresponding cut in the broadcast erasure network. First, we note that the corresponding cut in the broadcast erasure network is fundamental by a simple generalization of Lemma 13 to networks comprised of arbitrary broadcast channels. The value of the cut in the broadcast network and the polymatroidal network are within a factor $\psi(\epsilon, d)$ (since the achievable region and the cut of each broadcast erasure channel with feedback are within that factor by Lemma 18).

- Thus the gap between the achievable region and the cut is comprised of two parts, the $\psi(\epsilon, d)$ loss due the local channels and the factor $\kappa \log ^{3}(k+1)$ routing loss in polymatroidal networks with symmetric demands.

\section{CONCLUSION}

It is intriguing that the kind of symmetry that allows results suggesting the closeness of flow and edge-cuts also leads to the near-fundamentality of such edge-cuts. It would be interesting to see whether there is a deeper explanation of this phenomenon.

\section{ACKNOWLEDGements}

Sudeep Kamath gratefully acknowledges research support from the ARO MURI grant W911NF- 08-1-0233, Tools for the Analysis and Design of Complex Multi-Scale Networks, from the NSF grant CNS- 0910702, from the NSF Science and Technology Center grant CCF-0939370, Science of Information, from Marvell Semiconductor Inc., and from the U.C. Discovery program. 


\section{REFERENCES}

[1] S. Kannan and P. Viswanath, "Capacity of multiple unicast in wireless networks: A polymatroidal approach," arXiv:1111.4768 [cs.IT], Nov. 2011.

[2] R. Ahlswede, N. Cai, S.-Y. Li, and R. Yeung, "Network information flow," IEEE Transactions on Information Theory, vol. 46, pp. 1204-1216, July 2000.

[3] N. Harvey, R. Kleinberg, and A. Lehman, "On the capacity of information networks," IEEE Transactions on Information Theory, vol. 52, pp. 2345-2364, June 2006.

[4] R. Dougherty, C. Freiling, and K. Zeger, "Linear network codes and systems of polynomial equations," IEEE Transactions on Information Theory, vol. 54, pp. 2303-2316, May 2008.

[5] R. Dougherty, C. Freiling, and K. Zeger, "Insufficiency of linear coding in network information flow," IEEE Transactions on Information Theory, vol. 51, pp. 2745-2759, August 2005.

[6] T. Chan and A. Grant, "Mission impossible: Computing the network coding capacity region," in Proc. of IEEE ISIT, (Toronto, Canada), July 2008.

[7] G. Kramer and S. Savari, "Edge-cut bounds on network coding rates," Journal of Network and Systems Management, vol. 14, pp. 49-67, March 2006.

[8] J. Chuzhoy and S. Khanna, "Polynomial flow-cut gaps and hardness of directed cut problems," in Proc. ACM STOC, pp. 179-188, 2007.

[9] A. Agarwal, N. Alon, and M. Charikar, "Improved approximation for directed cut problems," in Proc. of ACM STOC, pp. 671-680, 2007.

[10] F. Leighton and S. Rao, "An approximate max-flow min-cut theorem for uniform multicommodity flow problems with applications to approximation algorithms," in Proc. of 28th Annual Symposium on Foundations of Computer Science, (Los Alamitos, California), 1988.

[11] N. Linial, E. London, and Y. Rabinovich, "The geometry of graphs and some of its algorithmic applications," Combinatorica, vol. 15, no. 2, pp. 215-245, 1995.

[12] S. Arora, S. Rao, and U. Vazirani, "Expander flows, geometric embeddings, and graph partitionings," JACM, vol. 56, no. 2, 2009.

[13] Z. Li and B. Li, "Network coding: The case of multiple unicast sessions," in Proc. of the 42nd Allerton Annual Conference on Communication, Control and Computing, September 2004.

[14] N. Harvey, R. Kleinberg, and A. Lehman, "Comparing network coding with the multicommodity flow for the k-pairs communication problem," in MIT LCS, Tech.Rep. 964, September 2004.

[15] P. Klein, S. Plotkin, S. Rao, and E. Tardos, "Bounds on the max-flow min-cut ratio for directed multicommodity flows," J. Algorithms, no. 22, pp. 241-269, 1997.

[16] S. Kamath, D. Tse, and V. Anantharam, "Generalized network sharing outer bound and the two-unicast problem," in Proc. of International Symposium on Network Coding, (Beijing, China), July 2011.

[17] I. Wang, S. Kamath, and D. Tse, "Two unicast information flows over linear deterministic networks," in Proc. of IEEE ISIT, (St. Petersburg, Russia), August 2011.

[18] J. Naor and L. Zosin, "A 2-approximation algorithm for the directed multiway cut problem.," SIAM Journal on Computing, vol. 31, no. 2, pp. 477-482, 2001.

[19] C. Chekuri, S. Kannan, A. Raja, and P. Viswanath, "Multicommodity flows and cuts in polymatroidal networks," Proc. of ITCS, 2012, available online on arXiv:1110.6832 [cs.IT], Oct. 2011.

[20] S. Kannan, A. Raja, and P. Viswanath, "Local phy + global flow: A layering principle for wireless networks," in Proc. of IEEE ISIT, (Saint Petersburg, Russia), August 2011.

[21] S. Kannan and P. Viswanath, "Multiple-unicast in fading wireless networks: A separation scheme is approximately optimal," in Proc. of IEEE ISIT, (St. Petersburg, Russia), August 2011.

[22] S. Thakor, A. Grant, and T. Chan, "Network coding capacity: A functional dependence bound," in Proc. of IEEE ISIT, July 2009.

[23] A. Avestimehr, S. Diggavi, and D. Tse, "Wireless network information flow: A deterministic approach," IEEE Transactions on Information Theory, vol. 57, pp. 1872-1905, April 2011.

[24] E. Lawler and C. Martel, "Computing maximal polymatroidal network flows," Math. Oper. Res., vol. 7, August 1982.

[25] R. Hassin, "On network flows," Ph.D Dissertation, Yale University, 1978.

[26] A. Motahari, S. Gharan, and A. Khandani, "Real Interference Alignment with Real Numbers," arXiv:0908.1208 [cs.IT], Aug. 2009.

[27] O. Oyman, R. U. Nabar, H. Bolcskei, and A. Paulraj, "Tight lower bounds on the ergodic capacity of rayleigh fading mimo channels," in Proc. of IEEE Globecom, pp. 1172-1176, 2002.

[28] B. Nazer, M. Gastpar, S. Jafar, and S. Vishwanath, "Ergodic Interference Alignment," in Proc. of IEEE ISIT, (Seoul, South Korea), 2009.

[29] A. Dana, R. Gowaikar, R. Palanki, B. Hassibi, and M. Effros, "Capacity of Wireless Erasure Networks," IEEE Transactions on Information Theory, vol. 52, pp. 789-804, March 2006.

[30] D. Lun, M. Médard, R. Koetter, and M. Effros, "On coding for reliable communication over packet networks," Physical Communication, vol. 1, pp. 3-20, March 2008.

[31] L. Georgiadis and L. Tassiulas, "Broadcast erasure channel with feedback capacity and algorithms," in Proc. 5th Workshop on Net. Cod. Theory and Appl., pp. 54-61, June 2009.

[32] C. Wang, "Capacity of 1-to-k broadcast packet erasure channels with channel output feedback," in Proc. of the 48nd Allerton Annual Conference on Communication, Control and Computing, October 2010.

[33] M. Gatzianas, L. Georgiadis, and L. Tassiulas, "Multiuser broadcast erasure channel with feedback - capacity and algorithms," arXiv:1009.1254 [cs.IT], Sept. 2010. 